\title{
Spike Timing-Dependent Synaptic Depression in the In Vivo Barrel Cortex of the Rat
}

\author{
Vincent Jacob, ${ }^{1}$ Daniel J. Brasier, ${ }^{2}$ Irina Erchova, ${ }^{1}$ Dan Feldman, ${ }^{2,3}$ and Daniel E. Shulz ${ }^{1}$ \\ ${ }^{1}$ Unité de Neurosciences Intégratives et Computationnelles, Centre National de la Recherche Scientifique, 91198 Gif sur Yvette, France, and ${ }^{2}$ Neurosciences \\ Program and ${ }^{3}$ Neurobiology Section, Division of Biology, University of California, San Diego, La Jolla, California 92093-0357
}

\begin{abstract}
Spike timing-dependent plasticity (STDP) is a computationally powerful form of plasticity in which synapses are strengthened or weakened according to the temporal order and precise millisecond-scale delay between presynaptic and postsynaptic spiking activity. STDP is readily observed in vitro, but evidence for STDP in vivo is scarce. Here, we studied spike timing-dependent synaptic depression in single putative pyramidal neurons of the rat primary somatosensory cortex (S1) in vivo, using two techniques. First, we recorded extracellularly from layer 2/3 (L2/3) and L5 neurons, and paired spontaneous action potentials (postsynaptic spikes) with subsequent subthreshold deflection of one whisker (to drive presynaptic afferents to the recorded neuron) to produce "post-leading-pre" spike pairings at known delays. Short delay pairings $(<17 \mathrm{~ms})$ resulted in a significant decrease of the extracellular spiking response specific to the paired whisker, consistent with spike timing-dependent synaptic depression. Second, in whole-cell recordings from neurons in L2/3, we paired postsynaptic spikes elicited by direct-current injection with subthreshold whisker deflection to drive presynaptic afferents to the recorded neuron at precise temporal delays. Post-leading-pre pairing ( $<33 \mathrm{~ms}$ delay) decreased the slope and amplitude of the PSP evoked by the paired whisker, whereas "pre-leading-post" delays failed to produce depression, and sometimes produced potentiation of whiskerevoked PSPs. These results demonstrate that spike timing-dependent synaptic depression occurs in S1 in vivo, and is therefore a plausible plasticity mechanism in the sensory cortex.
\end{abstract}

Key words: spike-timing dependent plasticity; STDP; somatosensory cortex; plasticity; rat; synaptic depression; LTP; LTD; barrel

\section{Introduction}

Long-term potentiation (LTP) and long-term depression (LTD) of synaptic transmission are well characterized forms of plasticity that occur in diverse brain areas and may mediate activitydependent neuronal development and learning (Frégnac and Shulz, 1999; Abbott and Nelson, 2000; Feldman and Brecht, 2005). In vitro studies have shown that LTP and LTD can be induced by changes in the relative millisecond-scale timing of presynaptic and postsynaptic action potentials (spikes), termed spike timing-dependent plasticity (STDP) (Levy and Steward, 1983; Bell et al., 1997; Debanne et al., 1997; Markram et al., 1997; Bi and Poo, 1998; Nishiyama et al., 2000; Kobayashi and Poo, 2004; Wang et al., 2005). In STDP at excitatory synapses on neocortical pyramidal cells, when a presynaptic spike and its evoked

\footnotetext{
Received Jan. 17, 2006; revised Dec. 20, 2006; accepted Dec. 23, 2006.

This work was partially supported by Action Concerteé Incitative-Ministère de la Recherche et Nouvelles Technologies (France) and by National Institutes of Health Grant R01 NS046652. The stays of V.J. and D.E.S. at the University of California, San Diego, for performing the whole-cell patch experiments were partially supported by Centre National de la Recherche Scientifique and contracts from the European Commission numbers IST2001-34712 (SenseMaker) and FP6-015879 (FACETS). D.J.B. was supported by a Howard Hughes Medical Institute predoctoral fellowship. We thank Drs. Alain Destexhe and Yves Frégnac for helpful comments on this manuscript.

Correspondence should be addressed to Daniel E. Shulz, Unité de Neurosciences Intégratives et Computationnelles, Centre National de la Recherche Scientifique, 1 Avenue de la Terrasse, 91198 Gif sur Yvette, France. E-mail: shulz@iaf.cnrs-gifffr.

I. Erchova's present address: Institute for Adaptive and Neural Computation, School of Informatics, Edinburgh University, Edinburgh, UK.

DOI:10.1523/JNEUROSCI.4264-06.2007

Copyright $\odot 2007$ Society for Neuroscience $\quad$ 0270-6474/07/271271-14\$15.00/0
}

EPSP precede a postsynaptic spike by up to a few tens of milliseconds ("pre-leading-post"), synaptic potentiation is induced. Conversely, synaptic depression occurs when EPSPs follow postsynaptic spikes ("post-leading-pre") (Feldman, 2000; Sjöström et al., 2001; Froemke and Dan 2002; Sjöström and Nelson, 2002; Meliza and Dan, 2006).

Despite intensive study in vitro, it remains unresolved how or whether STDP is induced in mammalian cortex in vivo. STDP may differ in vivo from in vitro because of different spiking patterns, levels of inhibition, and neuromodulation, which can affect the amount, likelihood, and duration of STDP (Sourdet and Debanne, 1999; Froemke and Dan, 2002; Lisman and Spruston, 2005). STDP has been inferred to occur in the visual cortex in vivo based on receptive field changes that occur in response to precisely timed stimuli (Schuett et al., 2001, Yao and Dan, 2001, 2004; Fu et al., 2002; René et al., 2003). STDP has also been directly observed at the level of synaptic responses in the visual cortex in vivo (Meliza and Dan, 2006). However, evidence for STDP in other cortical areas in vivo is lacking.

Here, we studied whether STDP can be induced at the singlecell and synapse level in the primary somatosensory cortex (S1) in vivo. We focused on the synaptic depression (LTD) component of STDP, which is produced in layer 2/3 (L2/3) and L5 pyramidal cells in vitro when postsynaptic spikes lead presynaptic spikes by up to 20-50 ms (Markram et al., 1997; Feldman, 2000; Sjöström et al., 2001; Froemke and Dan, 2002). We tested for STDP-LTD using two protocols. The first was a backward pairing protocol 
during extracellular unit recording in which we paired spontaneously emitted (postsynaptic) spikes of L2/3 and L5 cells with subthreshold whisker stimulation to drive presynaptic firing at defined delays after the postsynaptic spike. The second approach used whole-cell patch recording from L2/3 neurons in vivo, and induced STDP by pairing postsynaptic spikes elicited by current injection with whisker deflections that activated presynaptic afferents at defined times relative to postsynaptic spikes. Results from both protocols showed that post-leading-pre pairing drives depression of whisker responses, indicating that STDP-LTD can occur in S1 in vivo. In contrast, pre-leading-post pairing drove more sporadic response potentiation.

Some results have been published previously in abstract form (Jacob and Shulz, 2003; Shulz et al., 2004).

\section{Materials and Methods}

\section{Extracellular backward-pairing experiments}

Surgical preparation. Extracellular experiments were performed at Unité de Neurosciences Intégratives et Computationnelles-Centre National de la Recherche Scientifique (UNIC-CNRS, Gif sur Yvette, France). Maintenance, manipulations and surgery were performed in conformity with national (JO 87-848) and European legislation (86/609/CEE) on animal experimentation and met National Institutes of Health standards.

Adult male Wistar albino rats $(300 \pm 40 \mathrm{~g} ; n=22)$ were anesthetized with urethane $(1.5 \mathrm{~g} / \mathrm{kg}$, i.p.) and received atropine methyl nitrate $(0.3$ $\mathrm{mg} / \mathrm{kg}$, i.m.) to reduce respiratory secretions. Supplementary urethane $(0.15 \mathrm{~g} / \mathrm{kg}$, i.p. $)$ was administered when necessary to maintain adequate anesthesia, as assessed by the absence of corneal reflex, lack of response to mild hindpaw pinch, and lack of vibrissae movement. The electrocardiogram was monitored throughout the experiment. Body temperature was maintained at $37^{\circ} \mathrm{C}$.

The animal was mounted in a stereotaxic frame and the left scalp and temporal muscle were retracted. A local anesthetic (xylocaine 1\%) was injected subcutaneously before skin incision and applied as a cream to the ears before introducing the ear bars. A $4 \times 4 \mathrm{~mm}$ craniotomy was made to expose the left posteromedial barrel subfield (posterior 0-4 $\mathrm{mm}$, lateral 4-8 $\mathrm{mm}$ from bregma) (Chapin and Lin, 1984). The dura was opened and the craniotomy was filled with agar. The skull was cemented to a metal bar rigidly fixed to the stereotaxic frame, which enhanced recording stability, and the right ear bar was removed to allow free access to the right mystacial pad. The snout was held rigidly by a modified head holder (Haidarliu, 1996).

Electrophysiological recordings. Neuronal activity was recorded extracellularly with tungsten electrodes (FHC, Bowdoinham, ME) (2-10 M $\Omega$ at $1 \mathrm{kHz}$ ) mounted on an electronically controlled microdrive (MO-81; Narishige, Tokyo, Japan). Signals were amplified (gain 10,000) and filtered for spiking activity $(0.3-3 \mathrm{kHz}$; MCP Plus 8; Alpha-Omega, Nazareth, Israel). For each recording site, one single unit was isolated using a template-matching spike sorter (MSD; Alpha-Omega). Action potential shape was continuously inspected to ensure that the same neuron was recorded throughout the protocol. The electrode was advanced at least $100 \mu \mathrm{m}$ between recording sites to avoid recording the same units. The electrode signal was stored on a digital tape recorder (DTR 1801; sampling frequency 12 or $24 \mathrm{kHz}$; Biologic, Claix, France). Spike-time acquisition at $4 \mathrm{kHz}$ and data processing were done with custom-made software (Elphy; G. Sadoc, UNIC-CNRS). No neurons were recorded at the cortical depth corresponding to layer 4 . We estimated the laminar position of the units by establishing a correspondence between depth within the electrode penetration and the cortical layers. This relation was based on the depths of cortical layers determined by microscopic inspection of cresyl violet-stained coronal brain sections $(80 \mu \mathrm{m})$ from 10 additional experiments in which electrolytic lesions (30 pulses of $200 \mathrm{~ms}$ and $10 \mu \mathrm{A}$ delivered at $0.3 \mathrm{~Hz}$ ) were made at known depths, $500 \mu \mathrm{m}$ apart. Based on this relationship, layer 4 extended from 440 to $860 \mu \mathrm{m}$. Fifteen units were classified as supragranular (L2-L3) and 24 units as infragranular (L5-L6) according to the recording depth with respect to L4. The laminar position of three cells could not be accurately defined. The deepest supragranular cell was recorded at $375 \mu \mathrm{m}$, and the uppermost infragranular cell was recorded at $974 \mu \mathrm{m}$, sufficiently above and below the respective L4 borders.

Whisker stimulation and backward pairing protocol. Once units were isolated, vibrissae were first manually deflected while monitoring extracellular spikes. For each unit, the principal whisker (PW) was defined as the whisker eliciting the maximal spike count $(0-100 \mathrm{~ms}$ poststimulus). Response latency was defined as the first bin in a peristimulus time histogram (PSTH, $1 \mathrm{~ms}$ bin width) exceeding the level of the spontaneous activity by at least 3 SDs and for which the sum of the responses at that bin and the following one exceeded the level of two times the spontaneous activity by 4 SDs. The PW and one adjacent whisker (AW) in the same row were chosen for computer-controlled stimulation in the backwardpairing experiment. The majority of recordings were from barrel columns corresponding to whisker rows $\mathrm{B}, \mathrm{C}$, and $\mathrm{D}$, and the adjacent whisker was equally chosen from anterior and posterior positions (see Results). The PW and AW were clipped to $10 \mathrm{~mm}$ length, and inserted into short polypropylene tubes glued on piezoelectric bimorphs (Polytec-PI, Pantin, France). Piezos were driven with RC-filtered (time constant, $2 \mathrm{~ms}$ ) voltage pulses of $20 \mathrm{~ms}$ duration to produce rostrocaudal deflection of $7-700 \mu \mathrm{m}$ at $\sim 5 \mathrm{~mm}$ from the follicle, with an initial velocity of $8-800 \%$ s (see Fig. $1 C$ ).

Before pairing, control responses to suprathreshold deflection of the AW and PW were first measured. The AW and PW were deflected in trains of five deflections each at $0.5 \mathrm{~Hz}$, performed in a pseudorandom order every $10 \mathrm{~s}$ (see Fig. 1C). This yielded a total of 80 deflections per whisker. Deflection amplitude (range, 35-700 $\mu \mathrm{m}$ ) was chosen for both the PW and the AW to elicit significant suprathreshold responses. Then, to determine the amplitude of AW deflection to be used to generate subthreshold responses during pairing, whisker deflection amplitude was gradually decreased until no suprathreshold response was elicited (see Fig. 1A). The highest subthreshold deflection amplitude of the AW was defined as the activation threshold of the cell (range, 7-420 $\mu \mathrm{m} ; 17.5$ $\mu \mathrm{m}$ in the example presented in Fig. $1 A$ ), and was used during pairing. Stimulating the AW at deflection threshold avoided whisker deflectioninduced action potentials, which in turn would have triggered new whisker deflections. During pairing (Fig. $1 D$ ), each spontaneously emitted action potential of the recorded cell triggered subthreshold deflection of the AW with an imposed fixed delay of $0,10,20$, or $30 \mathrm{~ms}$. Each pairing period consisted of 400 action potential-deflection associations unless stated otherwise. When the interval between spontaneous action potentials was shorter than $20 \mathrm{~ms}$, no deflection was delivered to the second action potential. In this way, the presynaptic action potential (and, presumably, the corresponding PSP) was systematically delayed with respect to the postsynaptic action potential. Effects of pairing were assessed in a postpairing test period identical to the pre-pairing control. The absolute timing of the pairing was determined as the sum of the delay imposed by the experimenter and the latency of the response to the adjacent whisker deflection before pairing (calculated on the control PSTH). Two pairings at different temporal delays were attempted on some cells, separated by $10 \mathrm{~min}$. The pre-pairing control period considered for statistical analysis immediately preceded each pairing.

Data analysis. For each baseline and test period, we calculated $1 \mathrm{~ms}$ bin PSTHs for deflections of the unpaired and paired whiskers. After subtraction of the spontaneous activity calculated over the $40 \mathrm{~ms}$ preceding the stimulus, the responses were integrated along 20-ms-long windows, starting from 0 to $50 \mathrm{~ms}$ after the deflection onset and moving by $10 \mathrm{~ms}$ steps. The integration window containing the largest proportion of the population response to whisker deflection was between 10 and $30 \mathrm{~ms}$ (60\% of the total response) and was used for subsequent quantification and statistical tests. Cells with no significant response in this window were excluded from the analysis ( 7 of 91 pairings). Only $2 \%$ of the total response to whisker deflections was contained between 0 and $10 \mathrm{~ms}$ after the stimulus, as expected outside of L4.

For each pairing protocol, we calculated an index of normalized response changes for both paired and unpaired whiskers: $\Delta R=\left(R_{\mathrm{aft}}-\right.$ $R_{\text {bef }} /\left(R_{\text {aft }}+R_{\text {bef }}\right)$, where $R_{\text {bef }}$ and $R_{\text {aft }}$ are the responses to the PW or the AW within an integration window of $20 \mathrm{~ms}$ before and after pairing, respectively. We eliminated from the analysis the protocols where $\left(R_{\mathrm{aft}}+\right.$ $R_{\text {bef }}$ ) was smaller than 0.16 action potential (a.p.)/stimulus (stim), a 
threshold value that corresponds to a probability of 0.01 that $\left(R_{\mathrm{aft}}+R_{\mathrm{bef}}\right)$ differs from 0 .

To avoid including significant modifications of response to the paired whisker that might in fact be caused by general changes in excitability after pairing, we calculated an index of differential modifications of the response between the paired and the unpaired whiskers: $\Delta R_{\text {diff }}=$ $\Delta R_{\mathrm{AW}}-\Delta R_{\mathrm{PW}}$. Statistical significance of the differential modifications was determined by comparing the following equation:

$$
\frac{p \times\left(E_{2}-E_{1}\right)-q \times\left(E_{4}-E_{3}\right)}{\sqrt{p^{2} \times\left(\frac{\sigma_{2}^{2}}{n_{2}}+\frac{\sigma_{1}{ }^{2}}{n_{1}}\right)+q^{2} \times\left(\frac{\sigma_{4}{ }^{2}}{n_{4}}+\frac{\sigma_{3}{ }^{2}}{n_{3}}\right)}}
$$

with a Student law with $\left(n_{1} n_{2}+n_{3} n_{4}-2\right)$ degrees of freedom, where $E_{1}$, $E_{2}, E_{3}, E_{4}$ are the mean, $\sigma_{1}{ }^{2}, \sigma_{2}{ }^{2}, \sigma_{3}{ }^{2}, \sigma_{4}{ }^{2}$ are the variance, and $n_{1}, n_{2}, n_{3}$, $n_{4}$ the number of elements of the spike count in response to deflection of the AW before pairing, the AW after pairing, the PW before pairing, and the PW after pairing, respectively. The constants $p$ and $q$ are $p=1 /(E 1+$ $E 2)$ and $q=1 /(E 3+E 4)$, respectively. The test is mathematically equivalent to an unpaired two-tailed Student's $t$ test applied on the variables $\Delta R_{\mathrm{AW}}$ and $\Delta R_{\mathrm{PW}}$. All descriptive statistics are given as mean $\pm \mathrm{SD}$ unless stated otherwise.

Occasionally, nonstationarities in ongoing activity preceding whisker deflection mislead the quantification of the response. To avoid including divergent values of the response in the analysis, we compared the distribution of action potential counts in the $40 \mathrm{~ms}$ time window preceding deflection to a Poisson process distribution with the same mean $\left(\chi^{2}\right.$ test). The trials in which the activity preceding whisker deflection exceeded the expected value of a fitted Poisson distribution with an error of $10 \%$ were excluded from analysis ( $1 \%$ of the trials).

Intracellular measurement of whisker-evoked subthreshold responses. To determine whether AW deflection at activation threshold actually elicited subthreshold postsynaptic potentials during pairing, intracellular recordings were made in the barrel cortices of five adult male Wistar rats (320-390 g). Anesthesia and surgery were identical to the extracellular recording protocol, except that the craniotomy was smaller, $\sim 1 \mathrm{~mm}^{2}$, located 2.5-4.0 $\mathrm{mm}$ posterior and 5.5-7 $0.0 \mathrm{~mm}$ lateral to bregma, and the ear bars remained fixed during the recording session.

Whisker-evoked postsynaptic potentials were measured intracellularly (Axoclamp 2B) using sharp micropipettes filled with $2 \mathrm{M}$ potassium acetate and $1 \%$ biocytin (impedance, $75-120 \mathrm{M} \Omega$ ). Recordings were done in current-clamp mode, and the bridge was balanced manually on-line ( $-40 \mathrm{pA}, 150 \mathrm{~ms}$ current pulse). Recorded data were low-pass filtered at $3 \mathrm{kHz}$ and digitized at $10 \mathrm{kHz}$.

During intracellular recordings, the PW and one or two AWs were alternatively deflected 80 times each at $0.5 \mathrm{~Hz}$. We repeated the same protocol while reducing the amplitude of PW and AW deflection until only subthreshold responses could be recorded (see Fig. $1 B$ ).

Fitting models of STDP. To consider how whisker-evoked presynaptic spikes interacted with complex spontaneous postsynaptic spike trains to produce STDP, we fitted different models of STDP integration across spike pairs (Song and Abbott, 2001; Froemke and Dan, 2002) to the modifications of the evoked response $\left(\Delta R_{\mathrm{AW}}\right)$ and to the differential modifications $\left(\Delta R_{\text {diff }}\right)$. The fits were done only on pairings with whisker deflection amplitudes $>150 \mu \mathrm{m}$, and with 400 whisker deflections.

For the $i$ th whisker deflection and the $j$ th postsynaptic action potential, with an interval of time $\Delta t_{i j}$, the response modification $\Delta W_{i j}$ follows the standard, first-order STDP function: if $\Delta t_{i j}>0, \Delta W_{i j}=A^{+} \times$ $\exp \left(-\left|\Delta t_{i j}\right| / \tau^{+}\right)$; if $\Delta t_{i j}<0, \Delta W_{i j}=A^{-} \times \exp \left(-\left|\Delta t_{i j}\right| / \tau^{-}\right)$. The fitted function contains four parameters $\left(A^{+}, A^{-}, \tau^{+}\right.$, and $\left.\tau^{-}\right)$. For simplifying the calculation, only the pairs separated by an interval of time shorter that $100 \mathrm{~ms}$ were taken into account. The contributions $\left(\Delta W_{i j}\right)$ of each pair of an action potential and a whisker deflection were combined from different second-order models in the literature. First we used the classical STDP model (Song et al., 2000; van Rossum et al., 2000) where the contributions of individual spike pairs are combined either additively (linear model, $\Delta W=\Sigma_{i j} \Delta W_{i j}$ ) or multiplicatively [independent model, $\left.1-\Delta W=\Pi_{i j}\left(1-\Delta W_{i j}\right)\right]$ to obtain the amount of plasticity predicted by the model $(\Delta W)$. We then applied the suppression model proposed by
Froemke and Dan (2002) with the contribution of each of the individual spike pairs being corrected by eligibility factors before combining them either additively or multiplicatively [in the previous formula, $\Delta W_{i j}$ is replaced by $\varepsilon^{\text {pre }} \times \varepsilon^{\text {post }} \times \Delta W_{i j}$, where $\varepsilon^{\text {pre }}=1-\exp \left(-\left|\Delta t_{i, i-1}\right| / \tau_{s}{ }^{\text {pre }}\right)$ and $\varepsilon^{\text {post }}=1-\exp \left(-\left|\Delta t_{j, j-1}\right| / \tau_{s}^{\text {post }}\right) ; \Delta t_{i, i-1}$ is the interval of time between the $(i-1)$ th and the $i$ th evoked activity, and $\Delta t_{j, j-1}$ the interval of time between the $(j-1)$ th and the $j$ th action potential].

The fits were done with an unconstrained nonlinear minimization algorithm applied on the sum of the squared differences between the model and the data. The fit was repeated 25-30 times with different initial conditions spanning from 5 to $500 \mathrm{~ms}$ for the time constant $\tau$, from 1 to 0.001 for the scaling factor $A$, and from 10 to $200 \mathrm{~ms}$ for the time constant of the suppression function, $\tau_{s .}$. The fit maximizing the explained variance (i.e., the largest $R^{2}$ ) was selected.

\section{Whole-cell patch experiments in vivo}

Surgical preparation. Whole-cell experiments were performed at University of California, San Diego (UCSD) and approved by the UCSD Institutional Animal Care and Use Committee. Long-Evans rats (age, 27-32 $\mathrm{d} ; 61-106 \mathrm{~g})$ were anesthetized with urethane $(1.5 \mathrm{~g} / \mathrm{kg}, 25 \%$ in lactated Ringer's solution, i.p.) and given lactated Ringer's solution (2 ml, i.p.). The scalp was anesthetized with lidocaine and retracted, and a head bolt was attached to the skull with dental cement. The skull was thinned in a $2 \times 2 \mathrm{~mm}$ region overlying $\mathrm{S} 1$, and an $\sim 1 \times 1 \mathrm{~mm}$ patch of bone was removed (centered $5.2 \mathrm{~mm}$ lateral, $2.5 \mathrm{~mm}$ caudal of bregma). This recording craniotomy was surrounded by a well of dental cement, which was filled with HEPES-buffered Ringer's solution containing the following (in mM): $126 \mathrm{NaCl}, 20$ HEPES, $2.5 \mathrm{KCl}, 2 \mathrm{CaCl}_{2}, 1.3 \mathrm{MgSO}_{4}, 14$ D-(+)-glucose, $\mathrm{pH} 7.3$, with $\mathrm{NaOH}$. The dura was left intact. A separate craniotomy was made $\sim 2 \mathrm{~mm}$ posterior to the first to hold an $\mathrm{Ag} / \mathrm{AgCl}$ pellet recording ground, which was placed on intact dura, moistened with HEPES-buffered Ringer's solution, and held in place with silicone elastomer (Kwik-Cast; WPI, Sarasota, FL).

Recording procedures. During recording, anesthesia was maintained with a supplemental doses of urethane ( $10 \%$ of original dose, i.p.). Supplemental urethane was given whenever limb withdrawal responses were present, whisker movements were observed, or breathing rate exceeded 120 breaths $/ \mathrm{min}$. Body temperature was maintained at $37^{\circ} \mathrm{C}$ with a feedback-controlled heating blanket.

Whisker deflection was performed using an array of nine independent, computer-controlled piezoelectric actuators (Piezo Systems, Cambridge, MA), attached to individual whiskers in a $3 \times 3$ array. Whiskers were deflected with calibrated, $2^{\circ}$ ramp-and-hold deflections (upward or caudal deflection, $4 \mathrm{~ms}$ ramp, $100 \mathrm{~ms}$ hold, applied 5-6.5 $\mathrm{mm}$ from the face).

Whole-cell recordings were made using blind patch techniques (Margrie et al., 2002). A small opening $(\sim 100 \times 200 \mu \mathrm{m})$ was made in the dura for each penetration. Standard borosilicate glass patch electrodes were used (3.0-5.5 M $\Omega$; tip diameter, 2.5-3.5 $\mu \mathrm{m}$ ). Electrodes were filled with internal solution containing the following (in mM): $116 \mathrm{~K}$ gluconate, $6 \mathrm{KCl}, 2 \mathrm{NaCl}, 20$ HEPES, 0.5 EGTA, 4-5 MgATP, $0.3 \mathrm{NaGTP}, 10$ phosphocreatine, $\mathrm{pH} 7.20-7.25,285-295 \mathrm{mOsm}$. The craniotomy was kept filled with HEPES-based Ringer's solution. High positive pressure (2.5 psi) was applied while passing through the pia. After transiting the pia ( $\sim 50-150 \mu \mathrm{m}$ below the cortical surface), seals were sought in voltageclamp mode using $5 \mathrm{mV}$ seal test pulses, and low, intermittent pressure (0.1-0.2 psi) while advancing the electrode in $2.5 \mu \mathrm{m}$ steps. Penetrations were oriented $20^{\circ}$ off the radial axis. "Strikes" onto putative neurons were recognized by a twofold increase in electrode resistance, accompanied by characteristic amplification of heartbeat/breathing artifact. After a strike, gigaohm seal formation ( $>2 \mathrm{G} \Omega$ ) was obtained by release of pressure, gentle suction, and application of $-70 \mathrm{mV}$ holding potential. Whole-cell configuration was attained by applying a slow ramp of suction.

Whisker responses were recorded in current-clamp mode [Axopatch$1 \mathrm{D}, 10 \times$ gain, $2 \mathrm{kHz}$ low-pass filter, digitized at $5 \mathrm{kHz}$ using a 12-bit National Instruments (Austin, TX) board]. The bridge was generally not balanced, and membrane potential $(\mathrm{Vm})$ measurements were adjusted for uncompensated series resistance (Rs) off-line. Vm was not adjusted for the liquid junction potential. Whisker deflection, data acquisition, and analysis were performed by custom-written routines in Igor (Wavemetrics, Lake 
Oswego, OR). Series and input resistance (Rin) were measured in each sweep by responses to a square hyperpolarizing current injection (40-50 pA).

STDP induction protocol. PW and AW whiskers were deflected in alternation (1-1.5 s interstimulus interval) during baseline and test periods to elicit whisker-evoked subthreshold PSPs (wPSPs). For STDP induction, AW deflection was interrupted, and PW deflection was paired with current injection through the recording electrode $(1.0 \pm 0.3 \mathrm{nA}, 15-30$ $\mathrm{ms})$ to induce a postsynaptic spike burst ( $1.5 \pm 0.7$ spikes) shortly before or after the PSP. One hundred pairings were performed, using the same interstimulus interval as during baseline. Spike bursts were used, rather than single spikes, because the relatively high series resistance in vivo prevented the rapid depolarization necessary to reliably elicit precisely timed single spikes. Pre-post spike delay $(\Delta t)$ was calculated as the mean time of all evoked spikes in the postsynaptic burst minus the onset latency of the wPSP (Feldman, 2000). In a minority of pairings (five pre-leadingpost pairings), AW deflection was continued during PW pairing, interleaved but unpaired with current injection. Results of these pairings were equivalent to the three pre-leading-post pairings in which AW deflection was suspended during pairing (unpaired $t$ test, $p>0.45$ ), and data were merged here.

After PW pairing, PW and AW were again deflected in alternation to measure changes in wPSPs. At least $2.5 \mathrm{~min}$ (50 repetitions of each whisker) of postpairing data were required for inclusion in analysis. In 15 of 36 neurons, only single pairings were performed. In 21 neurons, multiple (two or three) pairing periods, separated by $\geq 10 \mathrm{~min}$, were performed. Plasticity in later pairings was not significantly different from that in early pairings (PSP amplitude, $p>0.8$; PSP slope, $p>0.4$, unpaired $t$ test). Thus, each pairing was considered a separate data point. A pairing was accepted for analysis only if series resistance and input resistance varied by $<20 \%$ between baseline and test periods, if $\mathrm{Vm}$ varied by $<5 \mathrm{mV}$, and if the PSP slope and amplitude showed a coefficient of variation $(\mathrm{CV})<4$ during the baseline period.

For each pairing, the wPSP amplitude (5 ms window) and initial slope (first $3.5 \mathrm{~ms}$ of wPSP) were calculated for the period (2.5-5 min before pairing) and the test period (first $5 \mathrm{~min}$ after pairing). Statistical significance of plasticity was determined using the nonparametric Kolmogorov-Smirnoff test (K-S; significance threshold 0.01) comparing all responses in these intervals. Magnitude of plasticity was defined as $\left[\left(\right.\right.$ response $_{\text {test }}-$ response $\left._{\text {baseline }}\right) /$ response $\left._{\text {test }}\right]$, as standard in the in vitro literature (Feldman, 2000). All citations of plasticity magnitude in the text use this measure. Exclusively for the analyses in Figure 8 and supplemental Figure 2 (available at www.jneurosci.org as supplemental material), magnitude of plasticity was plotted as the index $\triangle$ PSPAmplitude and $\triangle$ PSPSlope, which are defined (as for extracellular backwards pairing experiments) as (response $\mathrm{e}_{\text {test }}-$ response $\left._{\text {baseline }}\right) /\left(\right.$ response $_{\text {test }}+$ response $_{\text {baseline }}$ ). All descriptive statistics are given as mean \pm SD unless stated otherwise.

\section{Results}

\section{Experiment 1: backward extracellular pairing}

The experimental strategy was to induce synaptic depression by temporally correlating spontaneous spikes of a recorded neuron (postsynaptic spikes) with temporally delayed presynaptic spikes generated by mechanical whisker deflection. AW deflections were used for pairing, because these are thought to activate crosscolumnar corticocortical synapses, which are thought to be highly plastic in the somatosensory cortex (Fox, 2002), and exhibit STDP in the visual cortex (Dan and Poo, 2004). For pairing, AW deflection amplitude was reduced to a level that was just subthreshold for evoking spikes in the postsynaptic cell; our hypothesis was that this subthreshold deflection evoked presynaptic, but not postsynaptic spikes. [This hypothesis was verified in intracellular recordings (see below).] Post-leading-pre spike pairing was then implemented by using spontaneous postsynaptic spikes to trigger AW deflection at a fixed delay (backward pairing). This fixed delay plus the latency for AW deflection to evoke presynaptic spikes (estimated by the latency for AW re- sponses at the recording site) should approximate the imposed post-pre spike delay $(\Delta t)$ during pairing. We recorded 42 neurons from L2/3, L5, L6 of the primary somatosensory cortex of 22 adult rats. Eighty-four backward-pairing protocols were completed.

\section{Response characteristics to single whisker deflection}

The frequency of whisker stimulation during pairing was dictated by the spontaneous activity of each cell (Fig. $1 D)$. The spontaneous activity was on average $3.7 \pm 4.7$ a.p./s for L2/3 cells and $6.1 \pm$ 5.4 a.p./s for L5-L6 cells (recorded from control trials in the $40 \mathrm{~ms}$ preceding the stimulation of the principal whisker).

During baseline and postpairing periods, the AW deflection amplitude was on average 1.26 times larger than the PW deflection amplitude ( $p<0.01$, paired $t$ test), to generate AW responses that were roughly the same magnitude as PW responses. The mean response to the baseline PW stimulation was $1.05 \pm$ 0.88 a.p./stim (recorded $0-60 \mathrm{~ms}$ after whisker deflection onset, averaged over 80 stimulations delivered at $0.5 \mathrm{~Hz}$ ). The mean response to the baseline AW stimulation was $0.92 \pm 0.95$ a.p./ stim. The average response latency of single-units was $12 \pm 5.4$ ms for PW deflection and $14 \pm 7.6 \mathrm{~ms}$ for AW deflection $(p<$ 0.05 , paired $t$ test), as expected in these cortical layers.

During pairing, AW deflection amplitude was reduced to just below the threshold for generating postsynaptic spikes. Thus, AW deflections during pairing did not increase firing over spontaneous rates (baseline, $5.4 \pm 5.8$ a.p./s; during pairing, $5.0 \pm$ 3.7 a.p./s; $p>0.4$, paired $t$ test, $n=44)$, suggesting that plasticity cannot be attributed to spike rate changes.

\section{Subthreshold AW deflections elicit PSPs in intracellularly recorded cells}

The goal of the backward-pairing protocol was to pair spontaneous postsynaptic spikes with AW deflections that elicited presynaptic, but not postsynaptic spikes in the recorded neuron. Thus, AW deflections during pairing should generate subthreshold PSPs in the recorded neuron. To determine the threshold amplitude of AW deflection that elicited subthreshold PSPs, we gradually diminished AW deflection amplitude until sensory-evoked spikes just disappeared (Fig. $1 A$ ). This was termed the "activation threshold". To determine whether whisker deflection at the activation threshold still produced a significant PSP, we performed in vivo control experiments with sharp intracellular electrodes. Responses to nine AWs were recorded in six cells. For each cell, we recorded subthreshold and suprathreshold responses while varying the amplitude of PW and AW deflections.

Figure $1 B$ shows the average Vm and suprathreshold activity (PSTH) in response to three different whisker deflection amplitudes. Activation threshold was determined from spiking responses, as described for extracellular recordings (Fig. 1A). In $50 \%$ of the cases, the stimulation at the activation threshold evoked a significant PSP. The probability of evoking a PSP at the activation threshold depended on whisker deflection amplitude at activation threshold: subthreshold deflections $>150 \mu \mathrm{m}$ always evoked a PSP, subthreshold deflections of $35 \mu \mathrm{m}$ evoked a PSP in $50 \%$ of cases, and subthreshold deflections of $17.5 \mu \mathrm{m}$ or less evoked no PSP. Thus, deflection amplitude at threshold for generating a spiking response was significantly correlated with the amplitude of the evoked PSP (Fig. $1 \mathrm{~B}$, bottom graph) (slope, $\left.p<0.025 ; r^{2}=0.56\right)$.

These results indicate that only in cells with activation thresholds $>150 \mu \mathrm{m}$ was there a high probability that subthreshold AW deflection indeed drives presynaptic spikes, and elicits a PSP (Fig. 
A

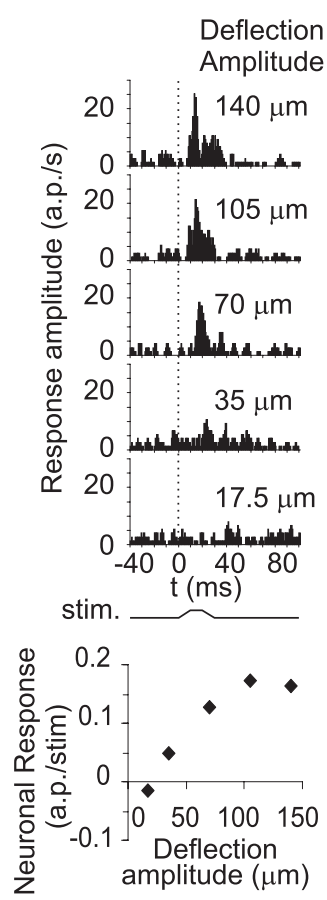

B
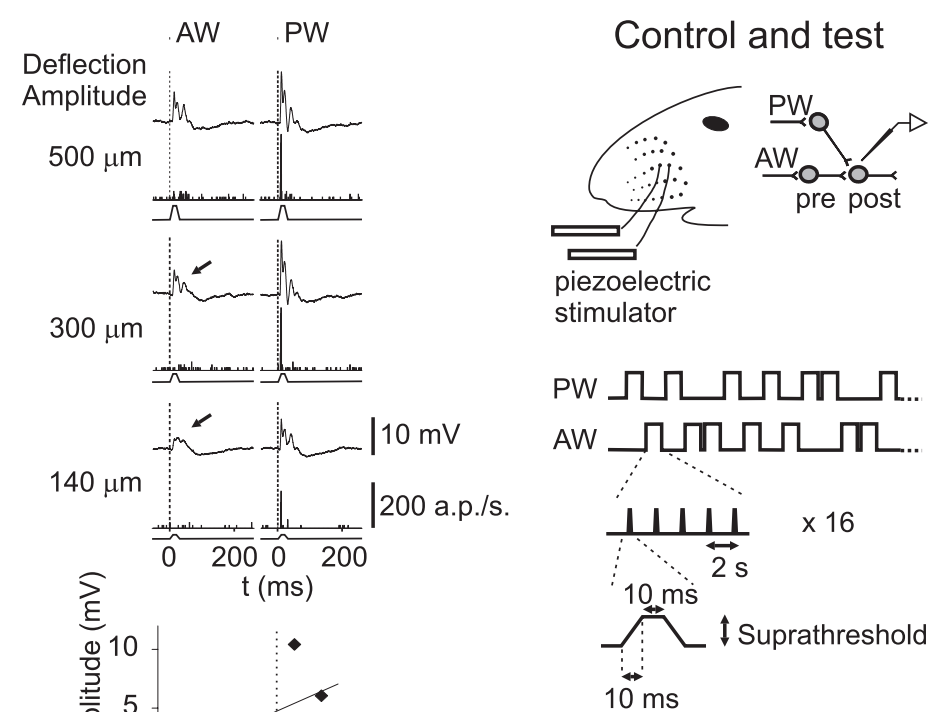

D
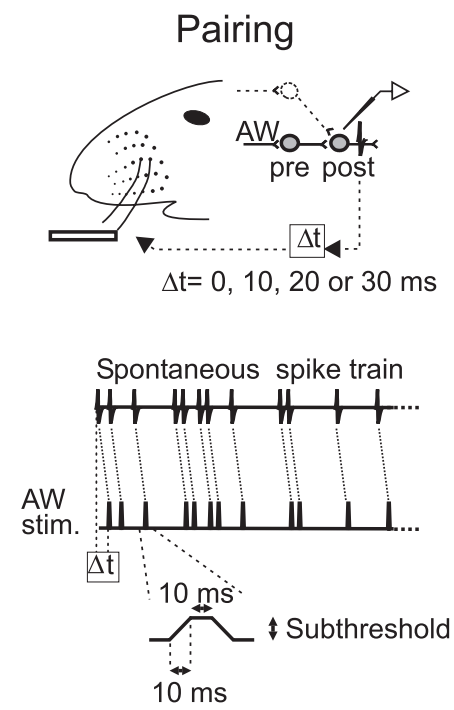

Figure 1. Experimental protocol for backward pairing. $A$, Peristimulus time histogram of responses to decreasing amplitudes of stimulation. The amplitude of the subthreshold stimulation used during pairing was $17.5 \mu \mathrm{m}$ in this example. The bottom graph shows the neuronal response integrated over $60 \mathrm{~ms}$ (mean spontaneous activity has been subtracted) as a function of the deflection amplitude. $\boldsymbol{B}$, Average membrane potential and corresponding PSTH in response to three deflection amplitudes. Bottom graph represents, for nine adjacent whiskers, the PSP amplitude as a function of the deflection amplitude at threshold. Whisker deflections $>150 \mu \mathrm{m}$ systematically produced large PSPs. C, During control and test, 16 trains of stimulation of the PW and of the AW were presented in alternation in a pseudorandom sequence. The trains of stimulation contained five deflections presented at $0.5 \mathrm{~Hz}$. The input waveform for each deflection was a $10 \mathrm{~ms}$ rostrocaudal movement followed by a $10 \mathrm{~ms}$ plateau and a ramp back to the rest position of the whisker. $\boldsymbol{D}$, During pairing, a spontaneously emitted action potential triggered a subthreshold deflection of the AW with a fixed delay $(0,10,20$, or $30 \mathrm{~ms})$. One pairing period contained 400 associations between an action potential and a stimulation of the AW.

$1 B$, dashed line, bottom graph). Thus, we assessed the effects of backwards pairing on cells that showed activation thresholds $>150 \mu \mathrm{m}$.

\section{Plasticity of responses induced by the backward pairing}

We assessed the effects of pairing on whisker responses by comparing the responses to PW and AW deflection before and after backward pairing consisting of 400 pairings of spontaneous postsynaptic spikes with subthreshold AW deflections. We calculated the indices $\Delta R_{\mathrm{AW}}$ and $\Delta R_{\mathrm{PW}}$ that quantify the response modifications for the AW (the paired whisker) and the PW (the unpaired whisker), respectively, and the index $\Delta R_{\text {diff }}$ that quantifies the differential changes of the response between the AW and the PW (see Materials and Methods).

Figure 2 shows the response of two cells to whisker deflection before and after pairing. The cell shown in Figure $2 A$, recorded in L5-L6 of the C2 barrel column, responded to AW (whisker C1) deflection with a latency of $12 \mathrm{~ms}$. Pairing consisted of 400 spontaneously emitted action potentials paired with a simultaneous ( 0 ms delay) subthreshold deflection of the whisker $\mathrm{Cl}$ (paired whisker). Because the post-pre delay during pairing $(\Delta t)$ was estimated as the sum of the delay of the onset of AW deflection after each spontaneous spike and the latency of the response to AW deflection, $\Delta t$ was $-12 \mathrm{~ms}$. After pairing, we observed a significant depression of the response to the paired whisker (response before and after pairing, 0.19 and 0.04 a.p./stim respectively; $p<0.05$ unpaired $t$ test). The response to the unpaired whisker showed small, nonsignificant facilitation (respectively 0.14 and 0.24 a.p./stim, $p>0.2$ ). The index $\Delta R_{\text {diff }}$ also revealed a significant differential depression (unpaired $t$ test, $p<0.02$ ), indicating that the response depression for the paired whisker was not caused by a general decrease in the excitability of the cell after pairing.

A second example of a differential depression of the response for an $\mathrm{L} 2 / 3$ cell in the $\mathrm{C} 4$ column is shown in Figure $2 B$. The cell responded to the AW (whisker C3) with a latency of $10 \mathrm{~ms}$. Four hundred spontaneously emitted action potentials were paired with a simultaneous ( $0 \mathrm{~ms}$ delay) subthreshold stimulation of the whisker C3. Thus, $\Delta t$ during pairing was $-10 \mathrm{~ms}$. The cell showed a $76 \%$ depression of the response to the paired whisker (respectively 0.46 and 0.11 a.p./stim before and after the pairing; unpaired $t$ test $p<0.001$ ), and no significant change in the response to the unpaired whisker (respectively 0.35 and 0.30 a.p./stim; $p>$ $0.5)$. Consequently, the index $\Delta R_{\text {diff }}$ showed a significant differential depression ( $p<0.05$, unpaired $t$ test).

In vitro studies in slices of the primary somatosensory cortex (Feldman, 2000) have shown that synaptic depression depends strongly on the precise timing of the pairing. We calculated $\Delta R_{\mathrm{AW}}$ and $\Delta R_{\mathrm{PW}}$ for an integration window of the PSTHs from 10 to 30 $\mathrm{ms}$ after the stimulus onset. This window captures most of the evoked response for the great majority of the cells, and particularly the initial phase of the response. In Figure 3, we plotted those indices against the $\Delta t$ during pairing for 19 protocols. For the statistical analysis, we divided experiments into broad, $10 \mathrm{~ms}$ bins of $\Delta t$, starting at $7 \mathrm{~ms}$ after the stimulus onset. This is the shortest latency that we could study because there is an uncompressible time necessary for the evoked response to reach the cortex after whisker deflection. We tested for changes in response to the PW 
A
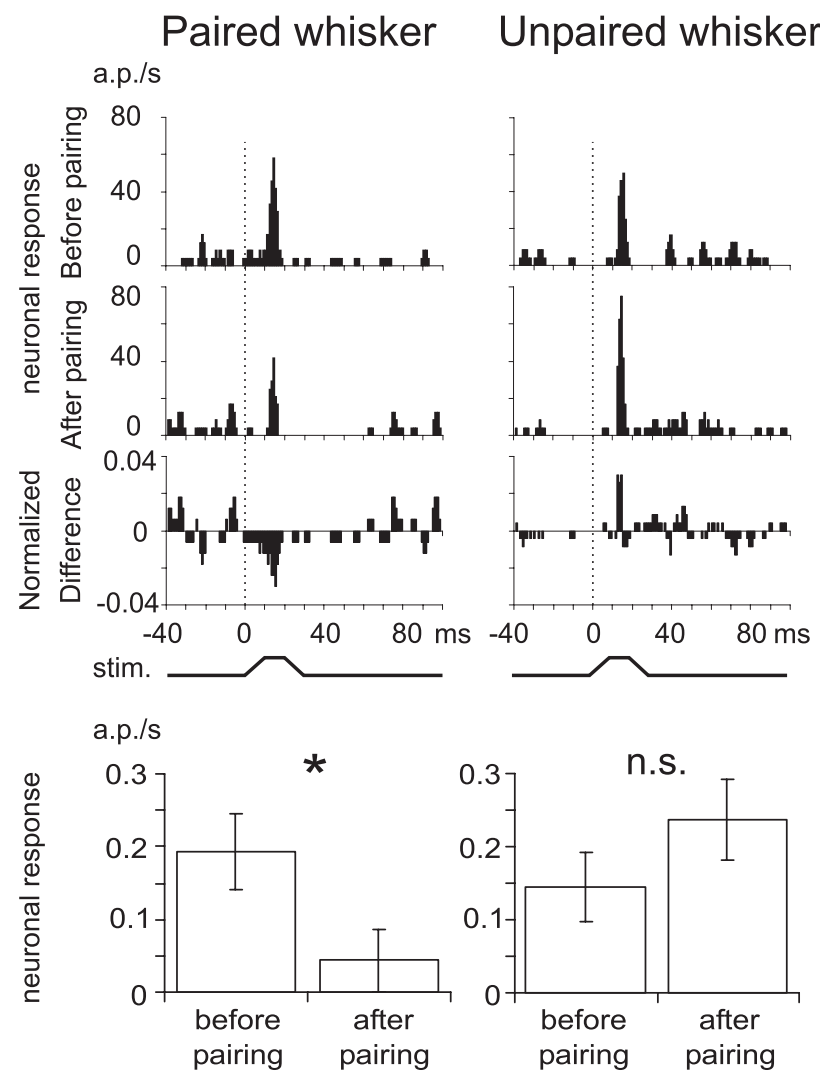

B
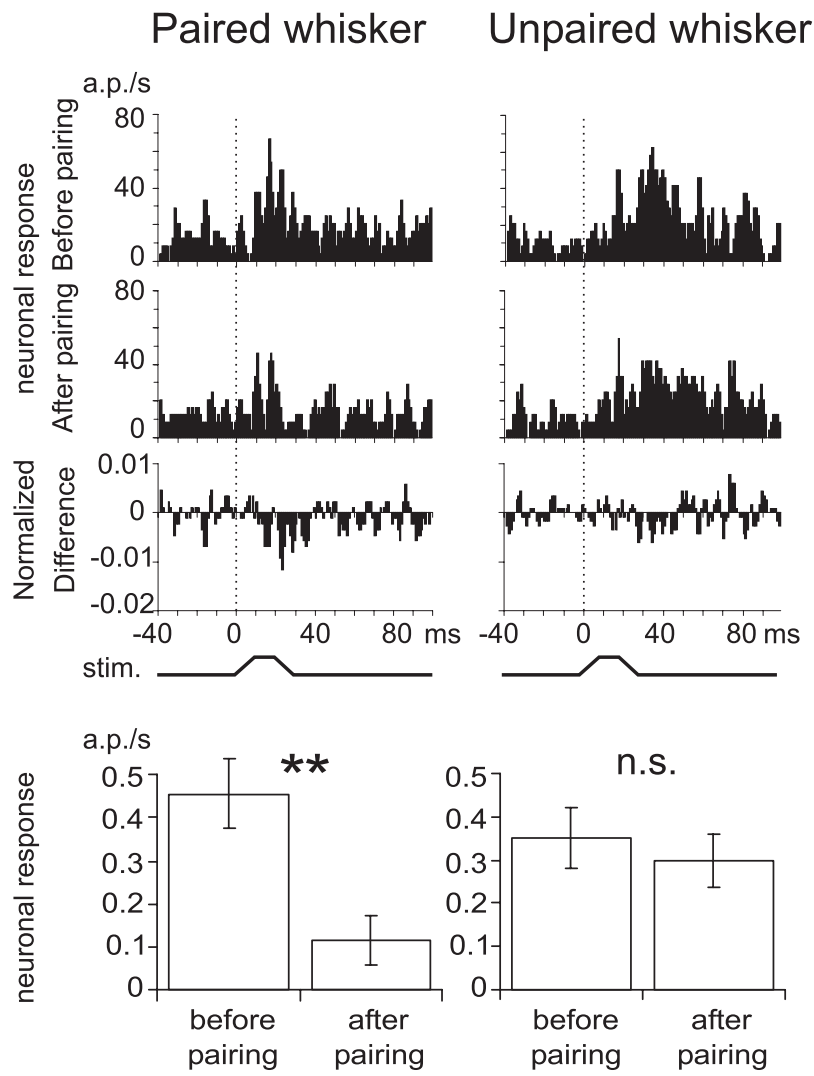

Figure 2. Differential depression of response by backward pairing. A, PSTHs of the response of a $\mathrm{L} 5$ - L6 single unit to control stimulations (before pairing) and to test stimulations (after pairing), as well as the normalized difference of responses for the paired whisker (whisker C1, left column) and the unpaired whisker (whisker C2, right column). Histograms show the response level before and after pairing. ${ }^{*} p<0.05$. B, Single unit recorded in L2/3. C 3 was chosen as the paired whisker (left column) and whisker ( 4 was chosen as the unpaired whisker (right column). Histograms show a significant $\left({ }^{* *} p<0.01\right)$ differential depression of the response to the paired whisker but not to the unpaired whisker.

and the $\mathrm{AW}\left(\Delta R_{\mathrm{AW}}\right.$ and $\left.\Delta R_{\mathrm{PW}}\right)$ with an unpaired Student's $t$ test. Depression of the response to the paired whisker, but not the unpaired whisker, was observed for the shortest time window of pairing delays ( $-17 \mathrm{~ms}<\Delta t \leq-7 \mathrm{~ms}$ ) across the population of cells $\left(\Delta R_{\mathrm{AW}}=-0.33, p<0.05 ; \Delta R_{\mathrm{PW}}=0.10\right.$, NS $)$. No significant response modifications for the other time windows were observed, indicating an extremely precise and short time window for efficient pre-post interactions. Depression of the response was still significant 5 min after the end of pairing $(p<0.01)$. This observation of timing-dependent depression supports the STDP model of plasticity in vivo.

The pairing was designed to induce plasticity of AW responses, but nonspecific modifications of responses cannot be excluded. To separate specific changes in AW responses from nonspecific changes that might affect evoked responses to both whiskers, we calculated the index $\Delta R_{\text {diff }}$ (see Materials and Methods). We assessed the statistical significance of the differential change $\Delta R_{\text {diff }}$ by performing a two-side paired $t$ test comparing $\Delta R_{\mathrm{AW}}$ and $\Delta R_{\mathrm{PW}}$. The short delay pairings (time window, $-17<$ $\Delta t \leq-7 \mathrm{~ms}$ ) resulted in a significant differential depression of the paired whisker response $\left(\Delta R_{\text {diff }}=-0.43 ; p<0.003 ; n=8\right)$ (Fig. 3). For all other delays, the pairing resulted in no significant changes. The unpaired whisker was always situated on the same row as the paired whisker and was equally distributed rostrally or caudally to the paired whisker ( 49 and 42 pairings, respectively). We found no significant difference in the level of differential depression for short delay pairings between rostral and caudal paired whiskers $(t$ test, $p>0.5)$.
During pairing, whisker responses were subthreshold and, thus, we do not know the type of synapses that are implicated in the pairing. Synapses sustaining the early or late phase of the response could be differently affected. This would result in a bias of response depression across the different phases of the PSTH. To estimate which phase of the response is changed by the pairing, we varied the delay of the window of integration of the response from stimulus onset (Fig. 4A). The response of the neurons was integrated over moving windows of $20 \mathrm{~ms}$, with a delay from the stimulus onset varying from 0 to $50 \mathrm{~ms}$. Because of the phasic nature of the cortical response, the majority of the neurons were included in the analysis for short integration time windows and fewer and fewer cells were included at longer delays. Figure $4 B$ shows the mean indices $\Delta R_{\mathrm{AW}}$ and $\Delta R_{\mathrm{PW}}$ calculated for the pairings with short $\Delta t$ (time window, $-17 \mathrm{~ms}<\Delta t \leq-7 \mathrm{~ms}$ ), plotted against the delay for response integration from the stimulus onset. The depression of the response was significant at 5 $(p<0.05)$ and $10(p<0.05)$ ms delays from the stimulus onset, and was maximal but not significant at $20 \mathrm{~ms}$. The very early and the late components of the response were not depressed.

\section{Influence of the amplitude of stimulation used during the pairing}

The amplitude of whisker deflection for the paired whisker during pairing was chosen to be at the activation threshold of the cell. This parameter changed from cell to cell and depended on intrinsic properties of the neuron and on the excitability state of the network. The results presented above are only for cells with acti- 

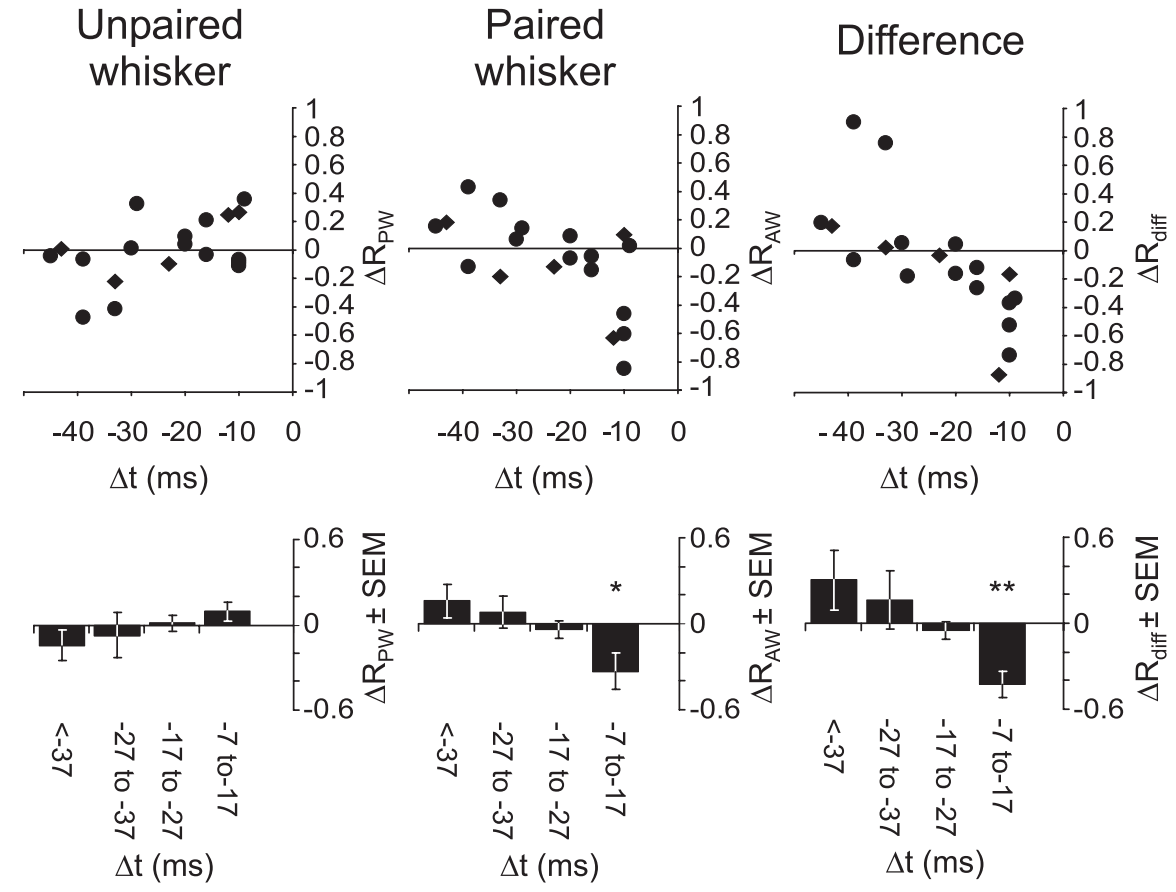

Figure 3. Specific depression for short delays of pairing. The response modification index for the unpaired whisker $\left(\Delta R_{\mathrm{PW}}\right)$, the paired whisker $\left(\Delta R_{\mathrm{AW}}\right)$, and the differential effect $\left(\Delta R_{\text {diff }}\right)$ is plotted against the delay $(\Delta t)$ of the pairing for $L 2 / 3$ (circles) and L5-L6 (diamonds) cells with deflection amplitude at an activation threshold larger than $150 \mu \mathrm{m}$. The delay has been corrected to take into account the latency of the cortical response. The histogram shows the mean response modification indices ( \pm SEM) for different delay windows. The depression is significant ( $t$ test, ${ }^{*} p<0.05 ;{ }^{* *} p<0.01$ ) only for pairings at a short delay window $(-17 \mathrm{~ms}<\Delta t \leq-7 \mathrm{~ms})$. The response to the unpaired whisker is not significantly modified at any of the tested delay time windows.

vation thresholds $>150$ microns in which there was a high probability of AW-evoked presynaptic spikes. In contrast, pairings with activation thresholds $<150$ microns did not produce significant plasticity (supplemental Fig. 1A, available at www. jneurosci.org as supplemental material). Across all activation thresholds, whisker deflection amplitude during pairing was significantly correlated with changes in response to the paired whisker for short-delay pairing $(\Delta t>-17 \mathrm{~ms})\left(\Delta R_{\mathrm{AW}}, p<0.005\right.$; $\Delta R_{\text {diff, }} p<10^{-6}$ ) (supplemental Fig. $1 B$, available at www. jneurosci.org as supplemental material). Significant plasticity was observed only for whisker deflection amplitude $>150 \mathrm{mi}-$ crons during pairing (compare Fig. 3, supplemental Fig. $1 \mathrm{~A}$, available at www.jneurosci.org as supplemental material). No significant correlation was found for long pairing delays, as expected because these delays do not drive plasticity (i.e., $\Delta t \leq-17$ $\mathrm{ms} ; p>0.05)$. These results indicate that two conditions have to be met for inducing depression of responses using our protocol. First, pairings should be at short delays, and second, the amplitude of whisker stimulation during pairing should be $>150$ $\mu \mathrm{m}$, which represents the threshold for consistently evoking PSPs (Figs. $1 B$, 3, supplemental Fig. $1 A$, available at www. jneurosci.org as supplemental material).

\section{Considering multiple interactions caused by the temporal stochastic nature of the pairing}

In vitro, STDP is often induced using low, fixed-frequency stimulation, which ensures that pairs of presynaptic or postsynaptic action potentials are substantially separated in time and do not significantly interact or interfere with one another. At the higher frequencies encountered in vivo, one postsynaptic action potential is temporally contiguous with more than one presynaptic action potential. Thus, multiple pairs of presynaptic and postsynaptic action potentials exist that predict concomitant induction of response potentiation and depression. Because our protocol pairing was dictated by spontaneously emitted action potentials, whisker deflection trains during the pairing were temporally irregular. To determine the impact of these temporal irregularities during pairing, we tested several established models of integration of the STDP rules over complex spike trains. We first fitted the data with an exponential function, which does not take into account the irregularity of the temporal structure of the spike trains during the pairing. We then fitted two models of STDP integration and compared them to the exponential fit: a model that assumes that each prepost spike pair is linearly integrated, and a model that includes nonlinear suppressive interaction between temporally adjacent spikes. The fits were done on 19 pairing protocols with amplitude of whisker deflection $>150 \mu \mathrm{m}$.

A two-parameter exponential curve between the response modifications $\left(\Delta R_{\mathrm{AW}}\right)$ and the delay of the pairing $(\Delta t)$ was fitted $\left(\mathrm{A}^{-}=-1.54 ; \tau^{-}=-6.9 \mathrm{~ms} ; R^{2}=0.32\right)$. The time constant $\tau$ appeared shorter than reported in vitro (Feldman, 2000; Celikel et al., 2004). We then fitted models of STDP function taking into account the multiple interactions caused by irregular trains of action potentials (Song and Abbott, 2001). The parameters resulting from the fits are reported in supplemental Table 1 (available at www.jneurosci.org as supplemental material). The classical STDP functions, in which the effect of each pair of action potentials was combined either linearly/ additively or independently/multiplicatively, resulted in a poorer correlation to the data than the exponential function $\left(R^{2}=0.24\right.$ and 0.25 , respectively). Finally, an additional factor has been included in the fit following the proposal that multiple nonlinear interactions between presynaptic and postsynaptic spikes can be accounted for by a suppressive eligibility factor that modulates the amount of change depending on the previous activity of the cell (Froemke and Dan, 2002). Both the additive $\left(R^{2}=0.33\right)$ and the multiplicative $\left(R^{2}=0.34\right)$ suppressive models including the eligibility factor to the response modifications resulted in similar correlation coefficients to the exponential fit.

The same analysis was performed on the index of differential modifications $\left(\Delta R_{\text {diff }}\right)$. The classical STDP function combined additively $\left(R^{2}=0.35\right)$ or multiplicatively $\left(R^{2}=0.38\right)$ resulted in similar correlation coefficients than the exponential curve $\left(R^{2}=\right.$ 0.38 ). However, the STDP function corrected by the eligibility factor resulted in a better correlation coefficient (additive model, $R^{2}=0.49$; multiplicative model, $R^{2}=0.53$ ) that explains roughly half of the variability of the response modifications.

\section{Experiment 2: STDP induction in whole-cell patch recordings in vivo}

In a second set of experiments, we used whole-cell recording in vivo to directly measure STDP of whisker-evoked subthreshold synaptic input. The strategy was to record wPSPs, and then to 


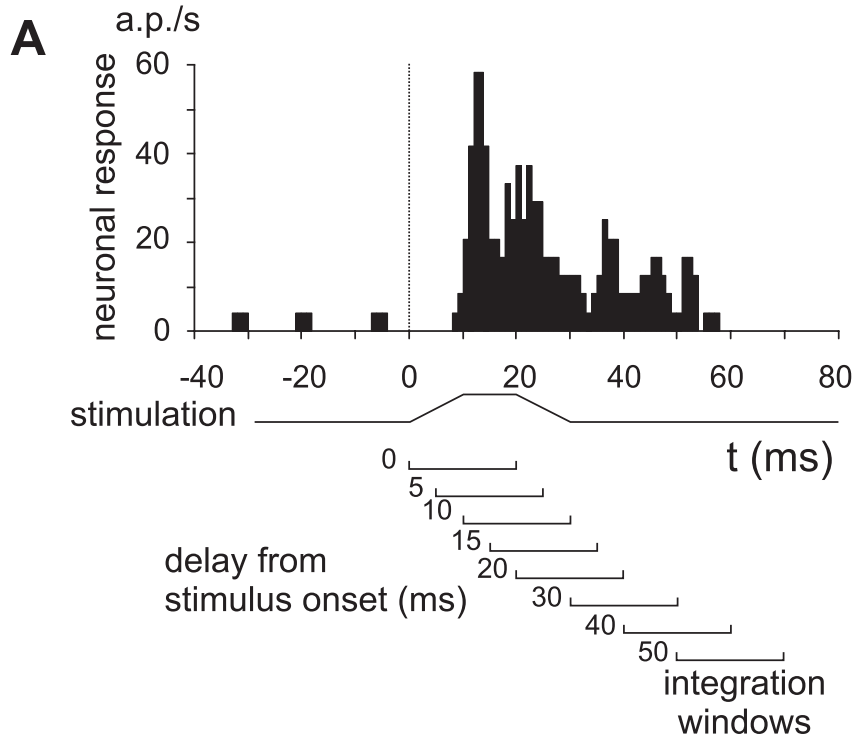

B

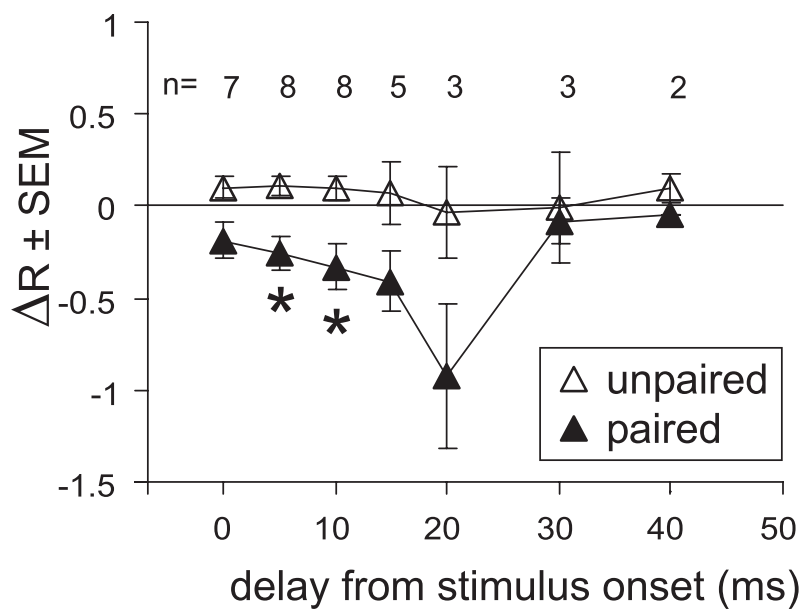

Figure 4. Analysis of response depression as a function of the delay from stimulus onset. $\boldsymbol{A}$, To assess the effect of pairing at different delays from stimulus onset, the responses to whisker stimulation were integrated on 20-ms-long time windows, the delay of which varied from 0 to $50 \mathrm{~ms}$ from stimulus onset. $\boldsymbol{B}$, The figure presents the mean response modifications for the paired ( $\Delta R_{\mathrm{AW}}$, close triangles) and the unpaired ( $\Delta R_{\mathrm{PW}}$, open triangles) whiskers at short-delay pairings ( $-17 \mathrm{~ms}<\Delta t \leq-7 \mathrm{~ms}$ ) as a function of the delay of the integration windows from stimulus onset, as defined in $A .{ }^{*} p<0.05$ between $\Delta R_{\mathrm{AW}}$ and $\Delta R_{\mathrm{PW}}$. Because of the phasic response of the neurons, the number of neurons taken into account for each integration window varies.

induce STDP by pairing wPSPs (which represent activation of presynaptic inputs to the recorded neuron) with postsynaptic spikes produced by direct current injection into the neuron. Unlike the extracellular backward-pairing experiment, this strategy allows for both post-leading-pre and pre-leading-post pairings, by varying the time of the current injection relative to the whisker deflection. It should also restrict plasticity to direct synaptic inputs onto the recorded neuron, because only that neuron spikes consistently relative to the whisker stimulus.

Thirty-six neurons were included in this study. Of these, 14 were regular spiking (RS) (Fig. $5 A$, top), six were intrinsically bursting (Fig. $5 A$, middle), and 16 were unclassified; unclassified neurons showed irregular firing patterns with no clear firing adaptation to $500 \mathrm{~ms}$ current injection. Fast-spiking neurons (putative interneurons) (Fig. 5A bottom) were excluded from analysis because of well known differences in plasticity rules at synapses on inhibitory versus excitatory neurons (Bell et al., 1997; Bi and Poo, 1998; Tzounopoulos et al., 2004). All cells were located 190-730 $\mu \mathrm{m}$ (median, $350 \mu \mathrm{m}$ ) below the pia, based on microdrive depth readings. These depths were shown previously to correspond to L2/3 (Celikel et al., 2004), although it is possible that a few of the deepest cells may have been in L4. We focused on L2/3 because L2/3 neurons exhibit well characterized STDP in vitro (Feldman, 2000) and have been suggested to do so in vivo (Celikel et al., 2004). Mean series resistance was $81 \mathrm{M} \Omega$, mean input resistance was $63 \mathrm{M} \Omega$, and mean resting potential was $-73 \pm 8 \mathrm{mV}(n=36)$.

\section{Induction of STDP in whole-cell recordings}

For each neuron, we first measured wPSPs evoked by each of nine whiskers in a $3 \times 3$ array. The PW was identified as the whisker evoking the wPSP with the steepest initial slope and shortest latency (Fig. 5 B, C) (Brecht et al., 2003). Deflections of the PW and one AW (typically the AW eliciting the largest wPSP) were then adjusted in amplitude and velocity to elicit roughly equal amplitude subthreshold wPSPs $(7.2 \pm 4.5$ and $5.7 \pm 3.7 \mathrm{mV}$ for the PW and the AW, respectively). We tried to avoid driving whiskerevoked postsynaptic spikes, but such spikes did occasionally occur when PSPs coincided with large spontaneous depolarizations, as occur normally in S1 in vivo (Brecht et al., 2003).

Baseline responses were measured to interleaved deflections of the PW and AW, with each whisker being deflected at 0.33-0.5 $\mathrm{Hz}$ in alternation. After a stable baseline ( $\sim 5 \mathrm{~min}, 50-100$ repetitions per whisker), we attempted to induce STDP by pairing deflection of the PW with current injection through the recording electrode to evoke one or more spikes in a short burst. The evoked spikes were timed to occur just before or after onset of the PW-evoked PSP. PW pairing was repeated 100 times, without altering stimulation rate, and the AW was not deflected (see Materials and Methods). The PW was chosen for pairing because PW responses in L2/3 neurons are mediated in large part by L4-toL2/3 synapses, which show robust STDP in vitro (Feldman, 2000). After PW pairing, responses to interleaved PW and AW responses were again measured.

Figure 6 shows an example of a post-leading-pre pairing in which postsynaptic spikes led the PW-evoked PSP by a mean of $-26 \mathrm{~ms}$ (Fig. 6B). Pairing caused the amplitude of the PWevoked PSP to decrease from $19.7 \pm 8.4 \mathrm{mV}$ during baseline to $15.4 \pm 10.1 \mathrm{mV}$ in the first $5 \mathrm{~min}$ postpairing (K-S, $p<0.05)$, and PSP initial slope to decrease from $1.8 \pm 0.9$ to $1.2 \pm 0.9 \mathrm{mV} / \mathrm{ms}$, $(\mathrm{K}-\mathrm{S}, p<0.0001)$. The depression lasted for the $10 \mathrm{~min}$ of postpairing recording and was specific to the paired PW whisker, because no significant change was observed for the unpaired AW whisker (baseline amplitude, $15.6 \pm 8.9$; postpairing, $15.4 \pm 7.6$ $\mathrm{mV}, \mathrm{K}-\mathrm{S}, p>0.7$; baseline slope, $1.5 \pm 0.7$; postpairing, $1.4 \pm 0.7$ $\mathrm{mV} / \mathrm{ms}, \mathrm{K}-\mathrm{S}, p>0.8)$.

In contrast to post-leading-pre pairing, pre-leading-post pairing often induced a mild response potentiation. Figure 7 shows one example of potentiation in an RS neuron (Fig. 7A) subjected to a $+15 \mathrm{~ms}$ delay pairing of the PW response (Fig. $7 B$ ). After pairing, the PW-evoked PSP increased from $6.8 \pm 0.5$ to $9.8 \pm 0.7$ $\mathrm{mV}(\mathrm{K}-\mathrm{S}, p<0.00001)$ and the PSP slope increased from $1.6 \pm$ 0.1 to $2.2 \pm 0.2 \mathrm{mV} / \mathrm{ms}$ postpairing (K-S, $p<0.00001$ ). Potentiation was accompanied by a change in the PSP amplitude for the unpaired $\mathrm{AW}(3.6 \pm 0.7 \mathrm{mV}$ during baseline, $4.9 \pm 0.6 \mathrm{mV}$ in the period from $0-5$ min postpairing period, K-S, $p<0.0005)$ that was transient and that rapidly returned to the baseline values. 
A
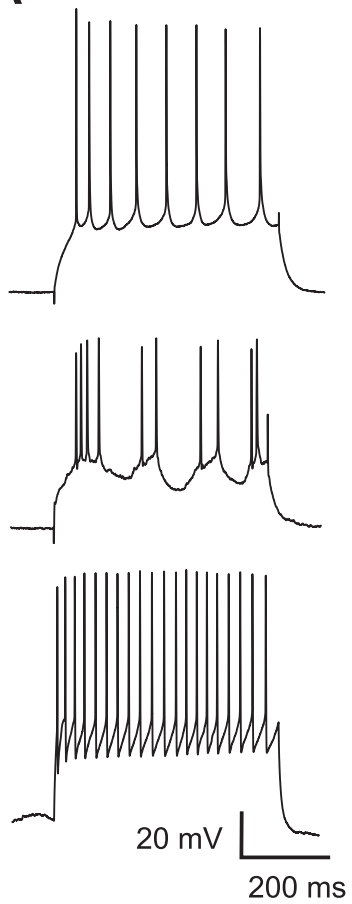

D
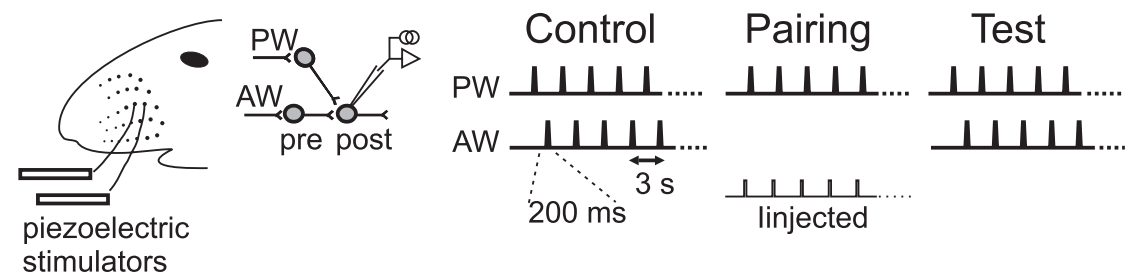

Figure 5. Experimental protocol for whole-cell induction of STDP in vivo. A, Examples of regular spiking (putative pyramidal neuron, top), intrinsic bursting (putative pyramidal neuron, middle), and fast spiking (putative inhibitory interneuron, bottom) firing patterns. $\boldsymbol{B}$, Subthreshold receptive field for one neuron. Each trace shows a wPSP elicited by the indicated whisker. The PW for this neuron was D2. C, Distribution of PSP latencies for the population of recorded neurons $(n=36)$ in response to stimulation of the PW (left) and the AW (right). D, STDP protocol for whole-cell experiments. During the control and the test periods, the PW and the AW were deflected alternately. During pairing, PW deflection was paired with current injection to elicit postsynaptic spikes.

-33 ms caused a $-19 \pm 5 \%$ (mean \pm SEM; $p<0.005 ; n=23)$ reduction in wPSP slope, and a $-17 \pm 3 \%(p<0.001$; $n=24)$ reduction in PSP amplitude. Depression did not occur for longer postleading-pre delays $(\leq-33 \mathrm{~ms}$ ) (wPSP slope, $-0.2 \pm 4.7 \%, p>0.7$; wPSP Amplitude, $-1.7 \pm 5 \%, p>0.4 ; n=5)$. Depression was also largely absent for preleading-post delays $(\Delta t>0 \mathrm{~ms})$. Instead, most cells with pre-leading-post pairings showed some degree of PSP potentiation, although the degree of potentiation was stronger for wPSP amplitude (23.2 \pm $10.1 \%$, mean \pm SEM) than wPSP slope $(3.3 \pm 8.4 \%)$ and did not attain significance across the population.

To characterize the mean timingdependence of synaptic depression, we fit an exponential function to the data points representing post-leading-pre pairing $(\Delta t<0 \mathrm{~ms}$ ) (Fig. 8). The resulting fit had a time constant of $19 \mathrm{~ms}$ for both PSP slope and PSP amplitude. This time window is shorter than that observed for STDP-LTD at L4-L2/3 synapses in vitro (Feldman, 2000).

The STDP rule in Figure 8 shows considerably more cell-to-cell variability than observed for regular spiking L2/3 pyramidal neurons in vitro (Feldman, 2000; Celikel et al., 2004). We examined several variables to determine the source of this variability. For pairings with $-33 \mathrm{~ms}<$ $\Delta t<-7 \mathrm{~ms}$, the measured $\Delta$ PSPSlope and $\Delta$ PSPAmplitude were not correlated with interstimulus interval during baseline or pairing $(0.33$ or $0.5 \mathrm{~Hz}$; unpaired $t$ test, $p>$ $0.09)$, the mean number of action potentials per burst during pairing $(p>0.2)$, baseline PSP initial slope $(p>0.8)$, baseline PSP amplitude $(p>0.2)$, or absolute input resistance $(p>0.1)$ or $\mathrm{Vm}(p>$

\section{The learning rule for spike timing-dependent synaptic depression in vivo}

The STDP learning rule across all pairings is shown in Figure 8. The magnitude of plasticity for each pairing is represented by the indices $\triangle$ PSPSlope and $\triangle$ PSPAmplitude, for consistency with backward pairing results. Most post-leading-pre pairings with pairing delay $(\Delta t)$ between -7 and -33 ms produced some amount of depression of PW-evoked PSP initial slope and amplitude, as reported by the $\Delta$ PSPSlope and $\Delta$ PSPAmplitude indices. Eleven of 23 cells with $\Delta t$ in this range showed significant depression of the PSP slope, and no cases of significant potentiation were observed. Conversely, four of eight pre-leading-post pairings showed significant potentiation of PSP amplitude, with no cases of significant depression observed. Although the potentiation side of this learning rule was not further explored, the overall shape of the learning rule is consistent with previous descriptions of STDP (Abbott and Nelson, 2000). Similar results were observed using the more conventional measure of the magnitude of plasticity, $\left(\right.$ response $_{\text {test }}-$ response $_{\text {baseline }}$ )/response baseline. Using $_{\text {b }}$ this metric, post-leading-pre pairings with $\Delta t$ between -7 and
$0.4)$. However, subclassifying neurons by firing patterns suggests that regular spiking cells may exhibit less variable STDP in vivo than intrinsically bursting or unclassified cells, leading to an STDP rule that resembles that observed in vitro (Fig. 8, bottom). On average, regular spiking cells showed a potentiation of wPSP amplitude, relative to baseline, of $38.9 \pm 8.8 \%$ (mean \pm SEM, $p<0.05 ; n=5$ ) during all pairings with $\Delta t>0$, and a depression of $-16.0 \pm 3.0 \%(p<0.01 ; n=7)$ for all pairings with $-33 \mathrm{~ms}<$ $\Delta t<-7 \mathrm{~ms}$. The changes in wPSP slope were in the expected direction $(+8.1 \%$ for pre-leading-post pairings and $-14.6 \%$ for postleading-pre pairings) but did not attain statistical significance.

\section{Nonassociative changes}

To ensure that changes in PW responses with STDP pairing were not caused by nonassociative changes (e.g., rundown of whisker responses caused by $0.5 \mathrm{~Hz}$ PW deflection), we conducted experiments in which the standard STDP protocol was run, except that current was not injected to elicit any spikes during the "pairing" period. In these cells $(n=5)$, we compared PW-evoked PSPs before and after the period that would normally correspond to 


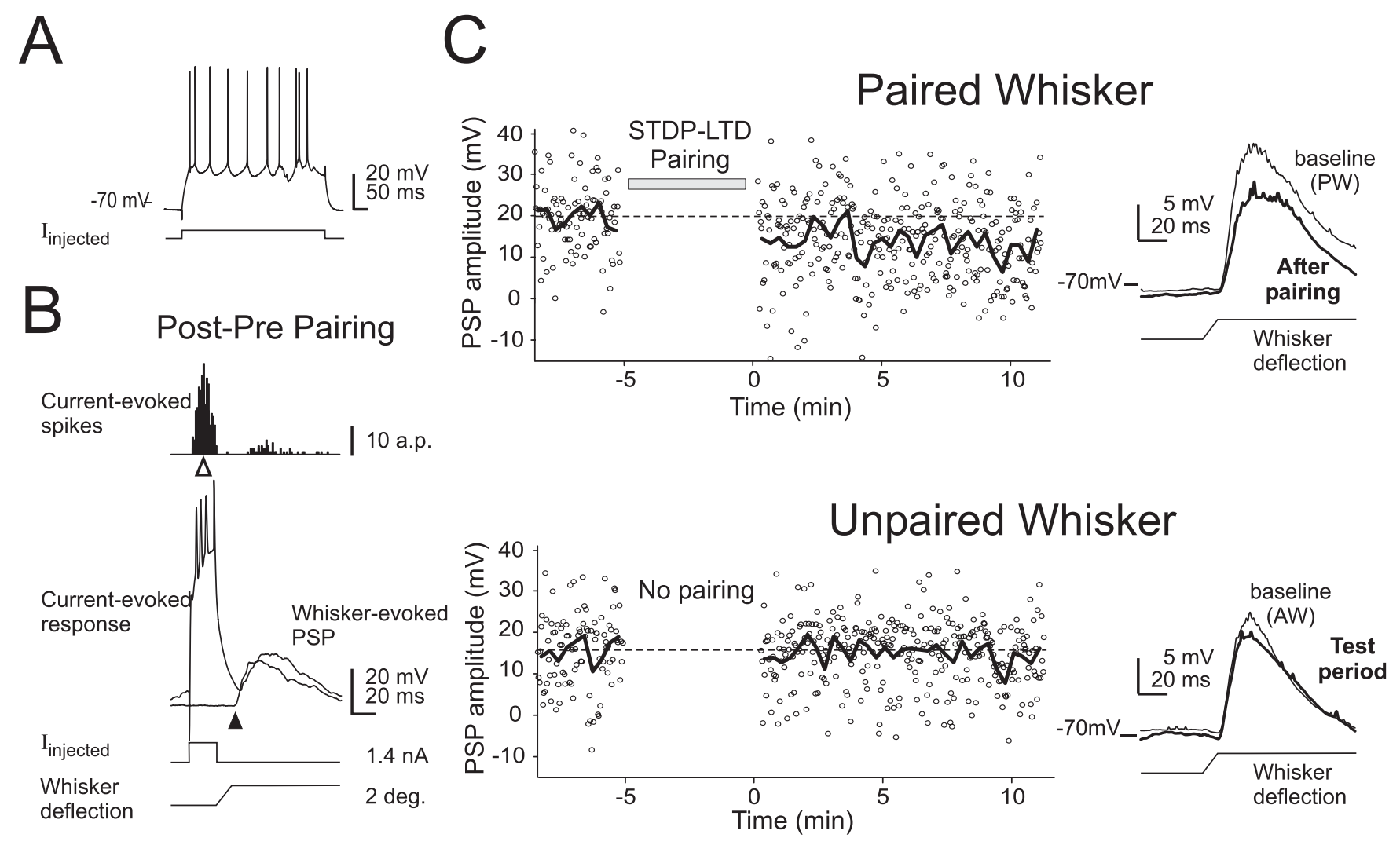

Figure 6. Induction of spike timing-dependent synaptic depression in a representative neuron. $\boldsymbol{A}$, Firing pattern in response to 500 ms current injection. $\boldsymbol{B}$, Pairing protocol in which currentevoked spikes preceded the PW-evoked PSP. Histogram, Distribution of times of all evoked spikes, relative to PSP onset (black arrowhead), during pairing. Mean $\Delta t$ for this cell was -26 ms (white arrowhead). C, Results of pairing. The amplitude of each wPSP during the experiment (small points) and the average of each 10 trials (thick line) for the paired PW (top) and unpaired AW (bottom) whiskers are shown. Right, wPSP averaged over 50 trials during baseline (thin line) and after pairing (thick line).

PW pairing. None of the cells subjected to this protocol showed significant changes in PSP amplitude or PSP slope. The average change in wPSP amplitude relative to baseline was $5.9 \pm 8.3 \%$ (mean \pm SEM, paired $t$ test, $p>0.5$ ). The average change in wPSP slope was $-2.9 \pm 5.7 \%(p>0.09)$. These changes, which represent the intrinsic, nonpairing-induced variability that occurs over this recording duration, are shown as gray regions in Figure 8. More than half of all real pairings with $-33 \mathrm{~ms}<\Delta \mathrm{t}<$ $-7 \mathrm{~ms}$ produced depression of $\mathrm{PW}$-evoked response slope or amplitude that was greater than this range of nonassociative variability (Fig. 8, top).

\section{Time course of pairing-induced plasticity}

We analyzed the time course of pairing-induced changes in paired, PW-evoked PSPs (Fig. 9). Experiments (pairings) were selected for time course analysis if (1) they passed standard stability tests for Rin, Rs, and Vm (see Materials and Methods), (2) they had at least 3 min of postpairing recording, and (3) they exhibited reliable baseline PSPs (CV for both PSP amplitude and slope $<4)$. Pairings were considered significant if either PSP slope or amplitude showed significant plasticity. Results for all pairings with $-33 \mathrm{~ms}<\Delta t<-7 \mathrm{~ms}$ show that the average effect was LTD, and that this LTD was maximal over 5 min of recording, and decayed somewhat between 5 and 10 min of recording. The subset of these pairings that showed significant plasticity (Fig. 8, black symbols) exhibited a similar time course of depression. For cells with $\Delta t>0 \mathrm{~ms}$ pairing, mean potentiation was apparent in the subset of cells with significant plasticity (corresponding to black symbols in Fig. 8), and remained roughly constant over the
8 min test period. When all cells subjected to $\Delta t>0 \mathrm{~ms}$ pairing were included, LTP was no longer apparent in the time course, consistent with the population data in Figure 8.

\section{Specificity of plasticity to paired PW versus unpaired AW pathways}

Only PW responses were paired with spiking in these experiments. We therefore examined whether spike timing-dependent depression was restricted to the paired PW pathway, or also occurred on the unpaired, AW pathway (supplemental Fig. 2, available at www.jneurosci.org as supplemental material). We computed the average $\Delta$ PSPAmplitude and $\Delta$ PSPSlope for all pairings for four broad subranges of $\Delta t(+10$ to $+20 \mathrm{~ms},-7$ to $-17 \mathrm{~ms},-17$ to $-33 \mathrm{~ms}$, and $<-43 \mathrm{~ms})$. Pairing significantly reduced $\Delta$ PSPAmplitude $(p<0.005)$ and $\Delta$ PSPSlope $(p<0.01)$ for -7 to $-17 \mathrm{~ms}$ and -17 to $-33 \mathrm{~ms}$ bins. Post-leading-pre pairings also reduced $\triangle$ PSPAmplitude and Slope for the unpaired AW, substantially in some neurons, but this achieved statistical significance across the population only for the amplitude and for -7 to $-17 \mathrm{~ms}$ bins $(p<0.05)$. Thus, the data indicate that plasticity may have spread partially to the unpaired pathway.

\section{Discussion}

In vitro, excitatory synapses on cortical pyramidal neurons exhibit STDP in which pre-leading-post spiking drives LTP, whereas post-leading-pre spiking drives LTD. How STDP is induced in vivo has been less clear, in part because cortical neurons in vivo experience a strong bombardment of synaptic input, including GABAergic input that has been predicted to alter or pre- 

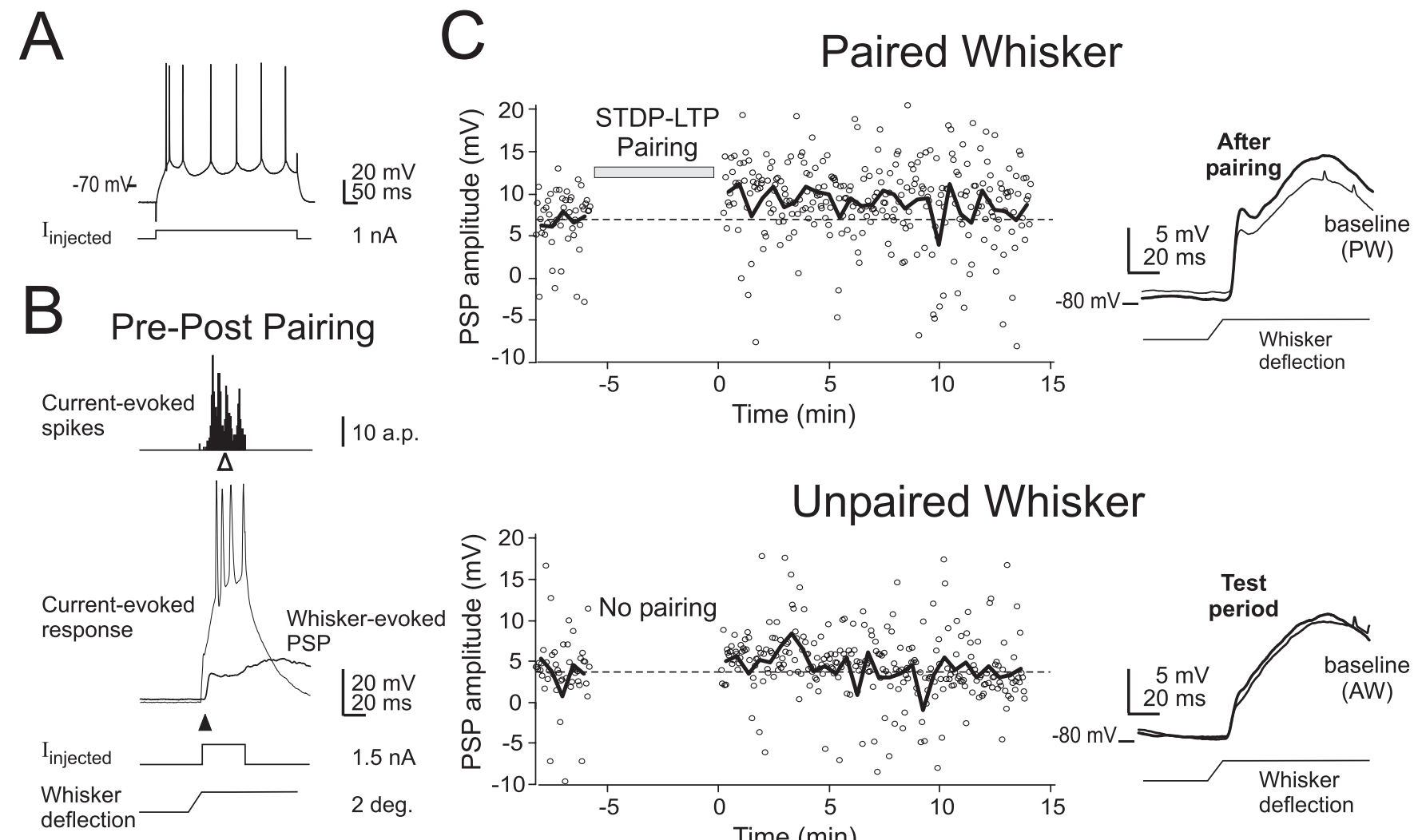

Figure 7. Induction of spike timing-dependent synaptic potentiation in a representative neuron. $\boldsymbol{A}$, Regular spiking pattern in response to $500 \mathrm{~ms}$ current injection. $\boldsymbol{B}$, Pairing protocol in which current-evoked postsynaptic spikes followed the PW-evoked PSP. Mean $\Delta t$ for this cell was $+15 \mathrm{~ms}$ (white arrowhead). C, Results of pairing on PW (paired) and AW (unpaired) wPSPs. Conventions are as in Figure 6.

vent STDP (Lisman and Spruston, 2005). Understanding how, and whether, STDP is induced at excitatory cortical synapses in vivo is critical because STDP at these synapses has been proposed to mediate experience-induced changes in sensory representations and sensory perception (Schuett et al., 2001; Yao and Dan, 2001; Fu et al., 2002; René et al., 2003; Celikel et al., 2004; Yao et al., 2004; Feldman and Brecht, 2005; Meliza and Dan, 2006). Whereas several previous studies have demonstrated STDP in the visual cortex in vivo (Schuett et al., 2001; Yao and Dan, 2001, Yao et al., 2004; René et al., 2003; Meliza and Dan, 2006), this is the first study to our knowledge that has tested STDP induction in S1 in vivo.

We observed significant spike timing-dependent depression of both whisker-evoked spikes (in the backwards pairing protocol) and synaptic responses (in the whole-cell protocol) in S1 pyramidal cells in response to post-leading-pre spike pairing in vivo. Depression was induced by a narrow range of post-leadingpre spike delays (up to 17-33 ms), consistent with STDP. Spike timing-dependent potentiation was tested only in a small number of experiments, and was occasionally observed, but was more sporadic.

\section{Spike timing-dependent depression of whisker-evoked spiking responses}

Plasticity of spiking responses in S1 was assessed using the backward pairing protocol in which a timing correlation was imposed between spontaneous postsynaptic action potentials and experimentally controlled whisker deflections to stimulate presynaptic afferents. Unlike previous plasticity studies in vivo (Frégnac et al., 1988; Schuett et al., 2001), no electrical or iontophoretic stimulation was applied during pairing, only mechanical whisker de- flection. Because spikes of the recorded neuron were not artificially triggered, whisker stimulation was constrained to follow postsynaptic spikes, so that only the depression side of the STDP curve (post-leading-pre pairings) could be investigated.

Results showed that backwards pairing drove significant timing-dependent depression of whisker-evoked spiking responses that was specific for the paired whisker, supporting the hypothesis that spike timing-dependent depression can be effectively induced by natural network activity patterns in vivo. As expected for STDP, plasticity was only induced when whisker deflection was likely to drive presynaptic spikes, which occurred with whisker deflection amplitude $>150 \mu \mathrm{m}$ during pairing. Recordings were made in both L2/3 and L5-L6, and both L2/3 and L5 synapses exhibit STDP in vitro (Markram et al., 1997; Feldman, 2000; Sjöström et al., 2001; Froemke and Dan, 2002). In our backward pairing study, although some L5-L6 cells showed significant response depression, the majority ( $83 \%$ ) of cells showing statistically significant depression were recorded in L2/3. This suggests that L2/3 may be more plastic than L5 in S1 in vivo, consistent with some previous studies of receptive field plasticity in S1 (Diamond et al., 1994; Huang et al., 1998). Alternatively, plasticity may have been more readily induced in L2/3 because whisker deflection amplitude at activation threshold tended to be higher in L2/3 than L5, reflecting the different activation properties of L2/3 and L5-L6 networks.

\section{Spike timing-dependent depression of whisker-evoked synaptic potentials}

Plasticity of subthreshold synaptic potentials was assessed in whole-cell experiments. Plasticity was induced by pairing 
whisker-evoked subthreshold PSPs, which reflect spiking activity of presynaptic afferents, with postsynaptic spikes elicited by direct current injection into the recorded neuron. Unlike backward pairing, this protocol allowed imposition of arbitrary pre-leading-post and post-leading-pre spike delays. Also unlike backward pairing, recordings were made in $\mathrm{L} 2 / 3$, and the PW was paired, rather than the AW. This was done because $\mathrm{PW}$ responses in $\mathrm{L} 2 / 3$ are primarily mediated by L4-L2/3 synapses (Armstrong-James et al., 1992), which exhibit well defined STDP in vitro, thus facilitating comparison between in vivo and in vitro STDP rules.

Results showed that post-leading-pre pairing $(-33 \leq \Delta t \leq-7 \mathrm{~ms})$ caused measurable depression of synaptic responses in most neurons (Fig. 8). Compared with STDP-LTD in pyramidal cells in vitro, cellto-cell variability was high. However, $70 \%$ of neurons showed some depression, $48 \%$ showed significant depression, and no neurons showed significant potentiation to this protocol. In contrast, when $\Delta t \approx$ $-50 \mathrm{~ms}$, or +10 to $+20 \mathrm{~ms}$, or when presynaptic stimulation occurred without evoked postsynaptic spiking, depression was not induced. Thus, we conclude that short post-leading-pre spike delays elicit spike timing-dependent depression of whisker-evoked synaptic responses in L2/3 pyramidal cells in vivo. (Recordings were likely from pyramidal cells because fastspiking neurons were excluded.) Whether plasticity occurred mainly at L4-L2/3 synapses, which classically drive PW responses in L2/3 neurons, is unknown.

Depression persisted for $5 \mathrm{~min}$, and in some cells for $12 \mathrm{~min}$, the longest duration of stable recording (Fig. 9). This duration is considerable given the rapid reversal of plasticity that can occur in vivo (Zhou et al., 2003). However, the duration of our recordings does not allow us to conclude whether LTD or a less persistent form of depression was induced.

Although these results indicate that post-leading-pre firing drives synaptic depression in vivo, whether pre-leading-post pairing reliably drives potentiation was less clear. Pre-leading-post pairing drove significant potentiation in some cells, particularly for PSP amplitude, but other neurons showed no plasticity or nonsignificant depression (Fig. 8). Significant LTP occurred only with pre-leading-post pairing, and not in the absence of postsynaptic spiking, or at other $\Delta t$ values. Although the potentiation side of the STDP rule was not explored in detail, the general shape of the learning rule (Fig. 8) is similar to STDP measured in vivo in the visual cortex (Meliza and Dan, 2006) and Xenopus tectum (Dan and Poo, 2006). Several factors could have reduced the likelihood of LTP in our experiments, including washout by intracellular dialysis (Malinow and Tsien, 1990; Feldman, 2000), a need for additional pairings, or the possibility that L4-L2/3 synapses may have saturated synaptic strength in vivo because of accrual of LTP by normal whisker use (Takahashi et al., 2003).

\section{PSP Amplitude}
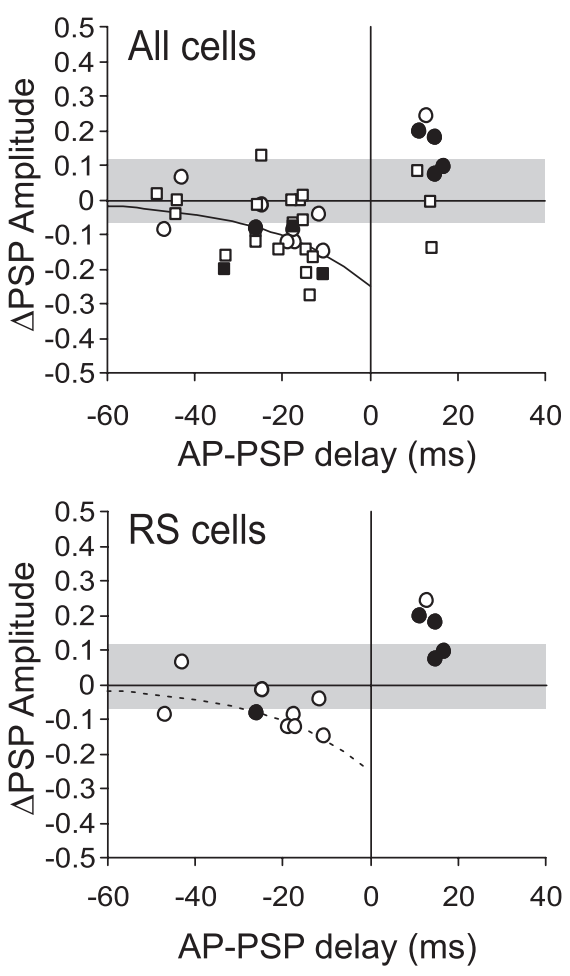

Figure 8. Learning rule for spike timing-dependent synaptic depression in $L 2 / 3$ in vivo. Pairing-induced changes in initial slope (left) and amplitude (right) of the wPSP as a function of delay between postsynaptic spikes and wPSP onset. Magnitude of plasticity is quantified using $\triangle$ PSPAmplitude and $\triangle$ PSPSlope indices (see Materials and Methods). Each (Kolmogorov-Smirnoff test, $p<0.01$ ). Gray region, Mean \pm SD for pseudopairing experiments in which no spikes were evoked during the pairing period. Pairings with $\Delta t<0$ were fitted with an exponential function. Bottom plots show results for RS cells only. The exponential fit computed on all pairings is reproduced on the RS plots (dashed line).

\section{Differences in STDP in vivo versus in vitro}

Synaptic depression appeared less stable in vivo (5-10 min, although full duration may have been masked by limitations in recording duration) than in vitro ( $>30 \mathrm{~min})$, consistent with the rapid reversal of LTP and LTD in active networks in vivo (Zhou et al., 2003). In addition, the range of post-leading-pre delays that drove synaptic depression was narrower in vivo (17 or $33 \mathrm{~ms}$ for backwards pairing and whole-cell experiments) than at L4-L2/3 synapses in vitro (50-100 ms) (Feldman, 2000; Celikel et al., 2004). This may reflect (1) restriction of STDP by network activity, GABAergic inhibition, or neuromodulation in vivo, (2) a contribution to plasticity in vivo by non-L4-L2/3 synapses with STDP rules with shorter LTD windows (Froemke et al., 2006), or (3) failure to detect modest LTD at longer spike timing delays because of high variability or lability of synaptic plasticity in vivo (Zhou et al., 2003).

In vitro, LTD can be synapse-specific or spread heterosynaptically (Bear and Abraham, 1996; Nishiyama et al., 1999). In our backwards-pairing experiments in vivo, depression was confined to the paired AW pathway; however, our whole-cell experiments showed modest spread of plasticity from paired (PW) to unpaired (AW) pathways. This discrepancy may be attributable to an inability to detect modest heterosynaptic spread by measuring sensory-evoked spikes, or may indicate that heterosynaptic spread only occurs from PW to AW pathways, not vice versa.

STPD induction in vivo occurs in the context of complex nat- 

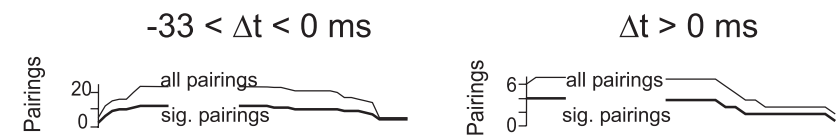

A1

B1
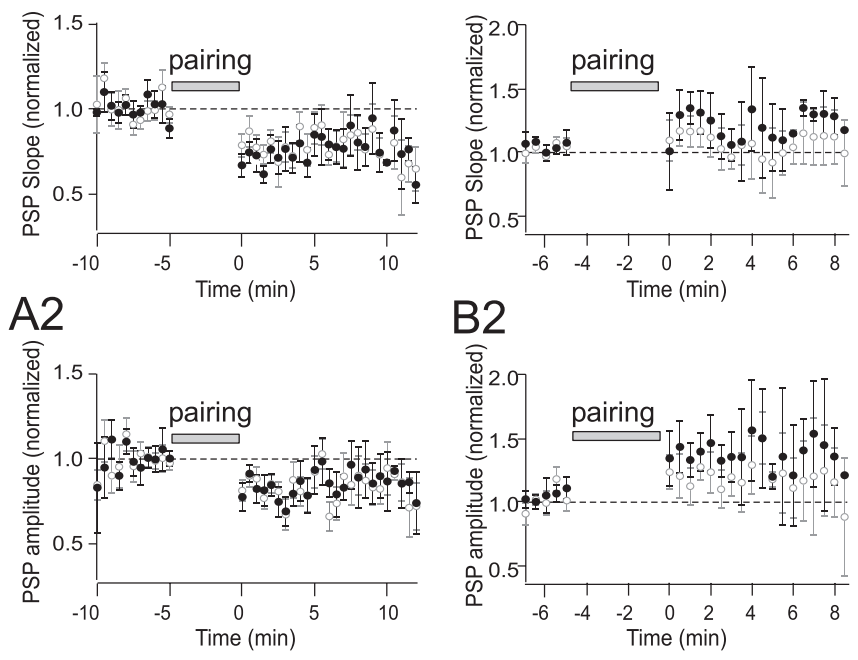

Figure 9. Mean time course of pairing-induced plasticity. The top plot shows the number of experiments contributing to each time point in the mean time course (data from a given pairing protocol was limited to the sweep range over which input resistance, series resistance, and Vm remained stable) (see Materials and Methods). A1, Pairing-evoked changes in wPSP slope, for pairings with $\Delta t$ between -7 and $-33 \mathrm{~ms}$. Open gray symbols, All experiments $(n=23$, each pairing was counted as one experiment). Filled symbols, Experiments that resulted in significant plasticity (i.e., the same experiments denoted by filled symbols in Fig. 8; $n=12$ ). Bars show SEM across experiments. A2, Pairing-evoked changes in WPSP amplitude for pairings with $\Delta t$ between -7 and $-33 \mathrm{~ms}$. Symbols as in $\mathbf{A 1}$. B1, Mean time course for wPSP slope for pairings with $\Delta t>0 \mathrm{~ms}$. Open symbols, All experiments $(n=7)$. Filled symbols, Experiments that resulted in significant plasticity $(n=4)$. $\boldsymbol{B}$, Mean time course for WPSP amplitude for pairings with $\Delta t>0 \mathrm{~ms}$. Symbols are as in $\boldsymbol{B}$.

ural spike trains, compared with the simple spike trains typically used to study STDP in vitro. High-frequency natural spike trains impose multiple interactions between presynaptic and postsynaptic spike pairs, each of which may contribute to synaptic depression or potentiation (Sjöström et al., 2001; Froemke and Dan, 2002; Kobayashi and Poo, 2004; Wang et al., 2005; Froemke et al., 2006). The integration of multiple interspike interactions is described by second-order STDP rules that are more complex than first-order rules describing plasticity from isolated pre-post spike pairs. We tested whether various second-order rules quantitatively predicted spike timing-dependent synaptic depression in vivo during backward pairing, which involved irregular, spontaneously emitted postsynaptic spike trains. Only a second-order rule in which the efficacy of each spike in producing synaptic modification is downregulated by previous spikes ["eligibility factor" (Froemke and Dan, 2002)] fit the data better than a firstorder rule. Even this rule, however, only partially explained the variance of plasticity that was observed. This may reflect intrinsically high variability of plasticity in vivo, or the inadequacy of known second-order rules.

\section{Conclusion}

These results show that spike timing-dependent synaptic depression can be induced in S1 in vivo by the conjunction of appropriately timed natural sensory stimuli and evoked or natural spikes. Depression is observed in both synaptic potentials and sensoryevoked spikes. Whether spike timing-dependent synaptic potentiation can also occur in vivo remains less clear. These results support the idea that STDP is a relevant learning rule in vivo and may contribute to map plasticity or learning.

\section{References}

Abbott LF, Nelson SB (2000) Synaptic plasticity: taming the beast. Nat Neurosci [Suppl]:1178-1183.

Armstrong-James M, Fox K, Das-Gupta A (1992) Flow of excitation within rat barrel cortex on striking a single vibrissa. J Neurophysiol 68:1345-1358.

Bear MF, Abraham WC (1996) Long-term depression in hippocampus. Annu Rev Neurosci 19:437-462.

Bell CC, Han VZ, Sugawara Y, Grant K (1997) Synaptic plasticity in a cerebellum-like structure depends on temporal order. Nature 387:278-281.

Bi GQ, Poo MM (1998) Synaptic modifications in cultured hippocampal neurons: dependence on spike timing, synaptic strength, and postsynaptic cell type. J Neurosci 18:10464-10472.

Brecht M, Roth A, Sakmann B (2003) Dynamic receptive fields of reconstructed pyramidal cells in layers 3 and 2 of rat somatosensory barrel cortex. J Physiol (Lond) 553:243-265.

Celikel T, Szostak VA, Feldman DE (2004) Modulation of spike timing by sensory deprivation during induction of cortical map plasticity. Nat Neurosci 7:534-541.

Chapin JK, Lin CS (1984) Mapping the body representation in the SI cortex of anesthetized and awake rats. J Comp Neurol 229:199-213.

Dan Y, Poo MM (2004) Spike timing-dependent plasticity of neural circuits. Neuron 44:23-30.

Dan Y, Poo MM (2006) Spike timing-dependent plasticity: from synapse to perception. Physiol Rev 86:1033-1048.

Debanne D, Gahwiler BH, Thompson SM (1997) Bidirectional associative plasticity of unitary CA3-CA1 EPSPs in the rat hippocampus in vitro. J Neurophysiol 77:2851-2855.

Diamond ME, Huang W, Ebner FF (1994) Laminar comparison of somatosensory cortical plasticity. Science 265:1885-1888.

Feldman DE (2000) Timing-based LTP and LTD at vertical inputs to layer II/III pyramidal cells in rat barrel cortex. Neuron 27:45-56.

Feldman DE, Brecht M (2005) Map plasticity in somatosensory cortex. Science 310:810-815.

Fox K (2002) Anatomical pathways and molecular mechanisms for plasticity in the barrel cortex. Neuroscience 111:799-814.

Frégnac Y, Shulz DE (1999) Activity-dependent regulation of receptive field properties of cat area 17 by supervised Hebbian learning. J Neurobiol 41:69-82.

Frégnac Y, Shulz DE, Thorpe S, Bienenstock E (1988) A cellular analogue of visual cortical plasticity. Nature 333:367-370.

Froemke RC, Dan Y (2002) Spike-timing-dependent synaptic modification induced by natural spike trains. Nature 416:433-438.

Froemke RC, Tsay IA, Raad M, Long JD, Dan Y (2006) Contribution of individual spikes in burst-induced long-term synaptic modifications. J Neurophysiol 95:1620-1629.

Fu YX, Djupsund K, Gao H, Hayden B, Shen K, Dan Y (2002) Temporal specificity in the cortical plasticity of visual space representation. Science 296:1999-2003.

Haidarliu S (1996) An anatomically adapted, injury-free headholder for Guinea pigs. Physiol Behav 60:111-114.

Huang W, Armstrong-James M, Rema V, Diamond ME, Ebner FF (1998) Contribution of supragranular layers to sensory processing and plasticity in adult rat barrel cortex. J Neurophysiol 80:3261-3271.

Jacob V, Shulz DE (2003) Spike-timing dependent depression in the barrel cortex of the adult rat. Soc Neurosci Abstr 29:58.14.

Kobayashi K, Poo MM (2004) Spike train timing-dependent associative modification of hippocampal CA3 recurrent synapses by mossy fibers. Neuron 41:445-454.

Levy WB, Steward O (1983) Temporal contiguity requirements for longterm associative potentiation/depression in the hippocampus. Neuroscience 8:791-797.

Lisman J, Spruston N (2005) Postsynaptic depolarization requirements for LTP and LTD: a critique of spike timing-dependent plasticity. Nat Neurosci 8:839-841.

Malinow R, Tsien RW (1990) Presynaptic enhancement shown by wholecell recordings of long-term potentiation in hippocampal slices. Nature 346:177-180.

Margrie TW, Brecht M, Sakmann B (2002) In vivo, low-resistance, whole- 
cell recordings from neurons in the anaesthetized and awake mammalian brain. Pflügers Arch 444:491-498.

Markram H, Lubke J, Frotscher M, Sakmann B (1997) Regulation of synaptic efficacy by coincidence of postsynaptic APs and EPSPs. Science 275:213-215.

Meliza CD, Dan Y (2006) Receptive-field modification in rat visual cortex induced by paired visual stimulation and single-cell spiking. Neuron 49:183-189.

Nishiyama M, Hong K, Mikoshiba K, Poo MM, Kato K (2000) Calcium stores regulate the polarity and input specificity of synaptic modification. Nature 408:584-588.

René A, Huguet N, Pananceau M, Grant K, Frégnac Y (2003) An in vivo generalisation of hebbian plasticity rules in adult visual cortex to multiple pre-post synaptic activity correlations. Soc Neurosci Abstr 29:266.17.

Schuett S, Bonhoeffer T, Hubener M (2001) Pairing-induced changes of orientation maps in cat visual cortex. Neuron 32:325-337.

Shulz DE, Brasier D, Feldman DE (2004) Spike-timing dependent plasticity investigated using whole-cell recording in rat somatosensory cortex (S1) in vivo. Soc Neurosci Abstr 30:57.1.

Sjöström PJ, Nelson SB (2002) Spike timing, calcium signals and synaptic plasticity. Curr Opin Neurobiol 12:305-314.

Sjöström PJ, Turrigiano GG, Nelson SB (2001) Rate, timing, and cooperativity jointly determine cortical synaptic plasticity. Neuron 32:1149-1164.
Song S, Abbott LF (2001) Cortical development and remapping through spike timing-dependent plasticity. Neuron 32:339-350.

Song S, Miller KD, Abbott LF (2000) Competitive Hebbian learning through spike-timing-dependent synaptic plasticity. Nat Neurosci 3:919-926.

Sourdet V, Debanne D (1999) The role of dendritic filtering in associative long-term synaptic plasticity. Learn Mem 6:10238-10248.

Takahashi T, Svoboda K, Malinow RT (2003) Experience strengthening transmission by driving AMPA receptors into synapses. Science 299:1585-1588.

Tzounopoulos T, Kim Y, Oertel D, Trussell LO (2004) Cell-specific, spike timing-dependent plasticities in the dorsal cochlear nucleus. Nat Neurosci 7:719-725.

van Rossum MC, Bi GQ, Turrigiano GG (2000) Stable Hebbian learning from spike timing-dependent plasticity. J Neurosci 20:8812-8821.

Wang HX, Gerkin RC, Nauen DW, Bi GQ (2005) Coactivation and timingdependent integration of synaptic potentiation and depression. Nat Neurosci 8:187-193.

Yao H, Dan Y (2001) Stimulus timing-dependent plasticity in cortical processing of orientation. Neuron 32:315-323.

Yao H, Shen Y, Dan Y (2004) Intracortical mechanism of stimulus-timingdependent plasticity in visual cortical orientation tuning. Proc Natl Acad Sci USA 101:5081-5086.

Zhou Q, Tao HW, Poo MM (2003) Reversal and stabilization of synaptic modifications in a developing visual system. Science 300:1953-1957. 